\title{
Expanding heterochromatin reveals discrete subtelomeric domains delimited by chromatin landscape transitions
}

\author{
Antoine Hocher, ${ }^{1,2}$ Myriam Ruault, ${ }^{1,2}$ Petra Kaferle, ${ }^{1,2}$ Marc Descrimes, ${ }^{1,2}$ \\ Mickaël Garnier, ${ }^{1,2}$ Antonin Morillon, ${ }^{1,2}$ and Angela Taddei ${ }^{1,2}$ \\ ${ }^{1}$ Institut Curie, PSL Research University, CNRS, UMR3664, F-75005 Paris, France; ${ }^{2}$ Sorbonne Université, UPMC Univ Paris 06 , \\ CNRS, UMR3664, F-75005 Paris, France
}

\begin{abstract}
The eukaryotic genome is divided into chromosomal domains of heterochromatin and euchromatin. Transcriptionally silent heterochromatin is found at subtelomeric regions, leading to the telomeric position effect (TPE) in yeast, fly, and human. Heterochromatin generally initiates and spreads from defined loci, and diverse mechanisms prevent the ectopic spread of heterochromatin into euchromatin. Here, we overexpressed the silencing factor Sir3 at varying levels in yeast and found that Sir3 spreads into extended silent domains (ESDs), eventually reaching saturation at subtelomeres. We observed the spread of Sir3 into subtelomeric domains associated with specific histone marks in wild-type cells, and stopping at zones of histone mark transitions including H3K79 trimethylation levels. Our study shows that the conserved H3K79 methyltransferase Dotl is essential in restricting Sir3 spread beyond ESDs, thus ensuring viability upon overexpression of Sir3. Last, our analyses of published data demonstrate how ESDs unveil uncharacterized discrete domains isolating structural and functional subtelomeric features from the rest of the genome. Our work offers a new approach on how to separate subtelomeres from the core chromosome.
\end{abstract}

[Supplemental material is available for this article.]

Heterochromatin classically designates chromosomal domains that remain condensed throughout the cell cycle (Heitz 1928). In contrast to gene-specific repressors, heterochromatin silences genes independently of their DNA sequence (Talbert and Henikoff 2006). Heterochromatin is prevalent in eukaryotic genomes and is key to processes including gene dosage compensation, cell differentiation, speciation, and genome stability (Grewal and Jia 2007).

Telomeres and a portion of subtelomeres are associated with heterochromatin in many species (Louis et al. 2014). Subtelomeres are genomic domains that are particularly difficult to define. Although they often exhibit structural and functional properties, such as the presence of specific gene families, chromatin marks or a relatively fast gene turnover, there is no strict definition that segregates all these properties between subtelomeres and the core genome (Louis et al. 2014).

Transcriptional silencing generally initiates at defined loci and propagates by self-recruitment mechanisms (Grunstein 1997; Hoppe et al. 2002; Grewal and Jia 2007; Gartenberg and Smith 2016). The coupling of histone modifying enzymes to nucleosomes allows the specific binding of silencing effectors and drives the formation of heterochromatin domains (Richards and Elgin 2002; Wang et al. 2016). However, the spread of heterochromatin must be limited to prevent encroaching on euchromatin (Donze and Kamakaka 2002).

In budding yeast, the silent information regulator (SIR) proteins-Sir2, Sir3, and Sir4-implement stable repression at the two cryptic mating-type loci (HML and $H M R$ ) and semistable repression of genes near telomeres (Rine and Herskowitz 1987;

Corresponding author: angela.taddei@curie.fr

Article published online before print. Article, supplemental material, and publication date are at http://www.genome.org/cgi/doi/10.1101/gr.236554.118.
Aparicio et al. 1991; Moazed et al. 1997; Rusche et al. 2003; Rudner et al. 2005; Grunstein and Gasser 2013; Gartenberg and Smith 2016). The SIR complex is recruited at these loci by a combination of specific DNA binding proteins that have functions outside silencing. At telomeres, the Repressor/Activator site binding Protein, Rap1, binds the degenerate telomeric sequence $\mathrm{TG}_{1-3}$ (Shampay et al. 1984) and recruits the SIR complex through direct interaction with Sir3 and Sir4. This recruitment is reinforced by additional interactions between Sir4 and the $\mathrm{Ku}$ heterodimers (Tsukamoto et al. 1997; Roy et al. 2004).

Once nucleated, the activity of Sir2, a conserved histone deacetylase, creates favorable binding sites for Sir3, which preferentially binds deacetylated H4K16. Iterative cycles of Sir2mediated histone deacetylation and Sir3 binding allow the selfpropagation of the SIR complex on chromatin until a barrier is eventually reached (Grunstein and Gasser 2013; Gartenberg and Smith 2016).

Boundaries restrict silent domains at the cryptic mating-type loci (Donze et al. 1999; Donze and Kamakaka 2001). A tRNA gene confines the Sir complex to HMR (Donze et al. 1999) while directional nucleation restricts silencing at HML (Bi et al. 1999). About half of subtelomeres have a $\mathrm{Y}^{\prime}$ middle repeat isolated from SIR spreading by the transcription factor Reb1. At these subtelomeres, adjacent internal TG repeats associated with the middle repetitive sequence core $\mathrm{X}$ can act as relays of silent chromatin propagation (Fourel et al. 1999, 2002; Pryde and Louis 1999; Thurtle and Rine 2014; Ellahi et al. 2015). Beyond these last

(c) 2018 Hocher et al. This article is distributed exclusively by Cold Spring Harbor Laboratory Press for the first six months after the full-issue publication date (see http://genome.cshlp.org/site/misc/terms.xhtml). After six months, it is available under a Creative Commons License (Attribution-NonCommercial 4.0 International), as described at http://creativecommons.org/licenses/ by-nc/4.0/. 
nucleation sites, the spread of silencing is rather limited ranging from hundreds of base pairs to a few kilobases, and no boundary has been identified so far. Furthermore, Sir3 was recently shown to extend inward chromosomes in G1-arrested cells (Mitsumori et al. 2016). Although numerous factors such as nuclear pore complex components and transcription factors display barrier properties in boundary assays, their physiological role in vivo remains to be explored (Oki et al. 2004). Last, the collective action of chromatin modifying enzymes implements chromatin states that potentially decrease Sir3 affinity for nucleosomes. In addition to the acetylation of H4K16 by the SAS-I complex, acetylation of histone H3 tails by Gcn5 and Elp3, methylation of H3K4 and H3K79, and H4K16ac-dependent incorporation of the H2A.Z histone variant were all proposed to contribute to limit Sir3 spreading at subtelomeres (Gartenberg and Smith 2016). In mutants lacking those enzymes or marks, the SIR complex propagates further away from the telomeres (Suka et al. 2002; Kristjuhan et al. 2003; Meneghini et al. 2003; Sperling and Grunstein 2009). However, the respective contribution of each mechanism to heterochromatin restriction and what further limits the spread of silencing in those mutants remains unknown.

A key parameter regulating heterochromatin dynamics, function, and spatial distribution is the concentration of silencing factors. For instance, increasing Sir3 dosage in budding yeast expands subtelomeric silent domains toward the chromosome core (Renauld et al. 1993) and increases telomere clustering (Ruault et al. 2011). However, extension of silencing domains was monitored at few subtelomeres, and the dose dependency of heterochromatin propagation remains qualitative (Renauld et al. 1993; Hecht et al. 1996; Strahl-Bolsinger et al. 1997; Katan-Khaykovich and Struhl 2005). Here we examine the impact of expressing the silencing factor Sir3 at varying levels genome-wide.

\section{Results}

Saturation of extended silent domains upon SIR3 overexpression

To systematically examine the impact of elevated Sir3 on the genome, we generated yeast strains that overexpress SIR3 at stable, different, and nonoverlapping levels. We replaced the endogenous SIR3 promoter with three different promoters, generating strains that produced $9 \times$-Sir3 ( $p S C A D H 1-S I R 3$, hereafter denominated "pADH-SIR3"), 16x-Sir3 (pSCTEF1-SIR3, "pTEF-SIR3"), and 29×-Sir3 ( $p$ SCTDH3-SIR3, " $p$ GPD-SIR3") as determined by Western blot (Supplemental Fig. S1A), and fluorescence quantification of live cells expressing Sir3-GFP (Fig. 1A; Supplemental Fig. S1B).
A

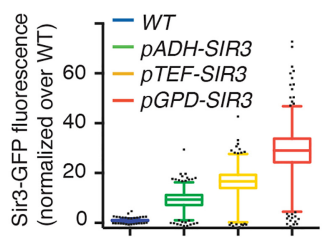

C

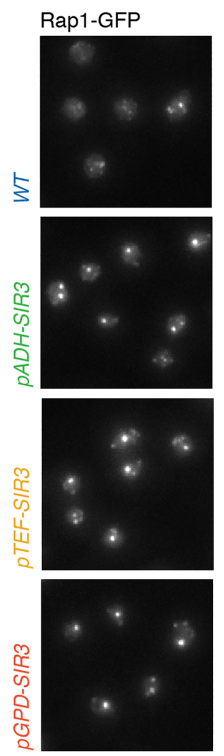

B

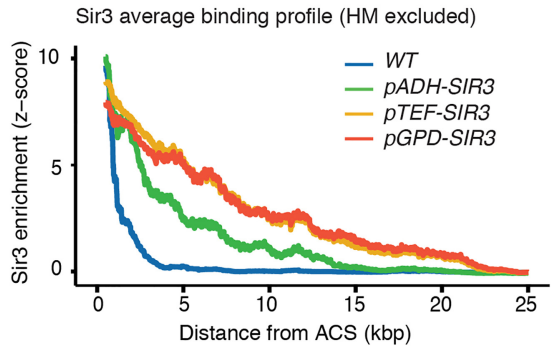

D Number of Rap1-GFP Foci per Cell

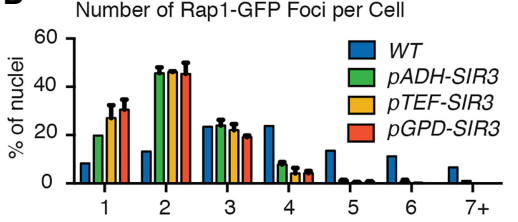

E
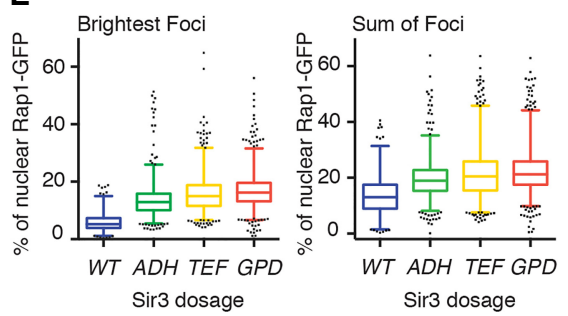

$\mathbf{F}$

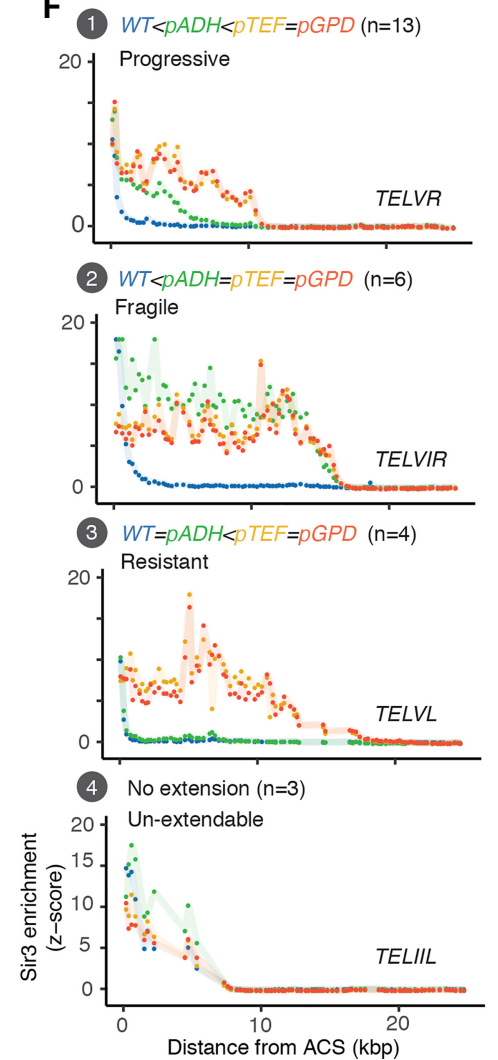

0.

(2) $W T<p A D H=D T E F=p G P D(\mathrm{n}=6)$ Fragil

:

$W T=p A D H<D T E F=p G P D \quad(n=4)$ Resistant

4 No extension $(n=3)$

Figure 1. Increasing Sir3 dosage leads to telomere clustering and Sir3 spreading saturation. (A) Quantification of Sir3 levels by integration of Sir3-GFP signal in strains expressing SIR3-GFP under different promoters as indicated. (B) ChIP-chip against Sir3 was carried in strains expressing SIR3 under different promoters as indicated. Moving average of Sir3 binding (block $=1000 \mathrm{bp}$, window $=10 \mathrm{bp}$ ) at telomeres (with the exception of TELIIIL and TELIIIR that contain $H M$ loci) as a function of distance from the ARS consensus sequence (ACS) within the last telomeric element. Enrichment is shown as the standardized IP over Input (see Methods). (C) Rap1-GFP foci grouping in strain differing for Sir3 levels. Cells were grown in YPD overnight, diluted to OD600 nm = 0.2 and imaged at OD600 nm =1. (D) Quantification of Rap1-GFP foci distribution in images from C. (E, left) Distribution of Rap1-GFP signal attributed to the brightest foci in each nucleus; (right) distribution of the relative amount of Rap1 measured within foci relative to total nuclear Rap1 signal. $(F)$ Stereotypical examples of Sir3 binding in function of Sir3 dosage. Enrichment corresponds to standardized Sir3 binding (Z-score). The number of subtelomeres within each group is shown in parentheses.

\section{Genome Research}

www.genome.org 
FACS profiles of wild-type and $p G P D$-SIR3 strains were largely similar, suggesting that the cell cycle was unaffected by overexpression of SIR3 (Supplemental Fig. S1C).

We probed genome-wide Sir3 binding by ChIP-chip using our antibody raised against the full-length native (untagged) protein (Ruault et al. 2011). We obtained a high signal to noise ratio (above 300 ) in the vicinity of known nucleation sites, namely TG repeats, ARS consensus sequence of the X-core elements (ACS), and the two cryptic mating-type loci, in agreement with previous studies (Sperling and Grunstein 2009; Radman-Livaja et al. 2011; Teytelman et al. 2013; Ellahi et al. 2015). To rule out potentially confounding effects due to well-documented ChIP artifacts, we compared the binding of Sir3 within subtelomeres to its binding at highly transcribed genes, a known source of artifactual signal (Park et al. 2013; Teytelman et al. 2013; Kasinathan et al. 2014). Finally, we compared Sir3 binding to that of the chromatin binding deficient mutant Sir3-A2Q as an additional control. In both cases, the signature of hyper-chipable loci was negligible compared to Sir3 binding signal at subtelomeres (Supplemental Fig. S1D,F), providing confidence in the integrity of our data.

On average in wild-type cells, we detected Sir3 binding up to $2.6 \mathrm{~kb}$ away from the last telomeric element (ACS), with some weaker signal at sites previously reported as secondary nucleation sites, consistent with previous studies (Lieb et al. 2001; Sperling and Grunstein 2009; Radman-Livaja et al. 2011; Ellahi et al. 2015). Elevation of Sir3 levels expanded the distance of Sir3 binding to $\sim 12 \mathrm{~kb}$ in $9 \times$ Sir 3 cells and up to $\sim 19 \mathrm{~kb}$ in $16 \times$ or $29 \times$ Sir 3 cells (Fig. 1B). Sir3 spreading was similar in $16 \times$ and $29 \times$ Sir3 cells. Sir3GFP nuclear background levels almost doubled in $29 \times$ Sir 3 compared to $16 \times$ Sir3 cells (Supplemental Fig. S1E), arguing that Sir3 binding to chromatin reached saturation. The constitutive overexpression of Sir3 submerged most of the secondary recruitment sites that were present in wild-type cells leading to the formation of extended continuous Sir3-bound domains at most subtelomeres. We observed that Sir3 binding increased at few euchromatic (nonsubtelomeric) sites upon Sir3 overexpression such as YAT1 or YFR017W (Supplemental Fig. S1F; Supplemental Table S1) but chose not to pursue this further, because in most cases changes in Sir3 binding were not associated with changes in gene expression in agreement with previous reports (Marchfelder et al. 2003).

In parallel, we monitored telomere foci in function of Sir3 concentration by live microscopy imaging of Rap1-GFP (Fig. 1C-E). In wild-type cells, telomeres cluster together in three to five foci located at the nuclear periphery (Gotta et al. 1996). However, upon Sir3 overexpression using the Gal1p promoter, most of the telomeres group together in the center of the nucleus (Ruault et al. 2011). In the range of Sir3 concentration probed, we observed that telomere clustering increased nonlinearly as a function of Sir3 levels, and this reached saturation at levels between 9-16×Sir3. Most cells (78\%) had at least three Rap1-GFP foci in the WT strain, whereas $64 \%-76 \%$ of cells had one or two foci in strains overexpressing Sir3 ninefold or more (Fig. 1D). Increased foci intensity paralleled the decrease in foci number (Fig. 1E, left), consistent with increased telomere grouping in cells overexpressing Sir3. Furthermore, the proportion of nuclear Rap1-GFP within foci increases from 13.6\% in WT cells to a maximum of $21.6 \%-22.2 \%$ for Sir3 dosage above 16-fold (Fig. 1E, right). Together, this suggests that not all telomeres are clustered within Rap1 foci in WT cells at a given time, and that elevated Sir3 levels increase the total number of telomeres within clusters, a process eventually reaching saturation. Thus, increased Sir3 dosage expands Sir3 genome binding and telomere clustering until they reach saturation between $9-16 \times$ Sir3 .
However, individual telomeres showed different stereotypical behaviors in response to increased Sir3 dosage. We classified telomeres based on their response to Sir3 dosage elevation (Methods). One example of each class is displayed in Figure 1F, and full data is shown in Supplemental Figure S1G. "Fragile" subtelomeres (6/ 26) displayed increased Sir3 spreading and plateaued at $9 \times \operatorname{Sir} 3$. "Progressive" subtelomeres (13/26) displayed gradually increased Sir3 spreading between $9-16 \times \operatorname{Sir} 3$ and then plateaued at $16 \times$ Sir3 . "Resistant" subtelomeres (4/26) displayed increased Sir3 spreading and plateaued at $16 \times$ Sir3. Finally, "insensitive" subtelomeres (3/26) did not expand in response to elevated Sir3 levels. The expanded Sir3 domains showed diverse lengths in all categories, ranging from 7 to $25 \mathrm{~kb}$ (HM excluded), independently of chromosomal arm length or middle repeat content (Supplemental Fig. S1H,I).

\section{Sir3 spreading extends silent domains}

Overexpression of SIR3 repressed subtelomeric transcription, as expected. Given that overexpression of the point mutant SIR3-A2Q, which leads to telomere clustering but only binds to telomeric repeats (Fig. 2A,B), did not affect global transcription of subtelomeres (Supplemental Fig. S2A), repression was attributed to Sir3 binding to chromatin and not clustering of telomeres. However, 22 genes showed transcriptional changes common to the overexpression of SIR3-A2Q and SIR3, that is, potentially caused by telomere clustering, including 20 euchromatic genes (Supplemental Fig. S2D). Those transcriptional changes could be the consequences of changes in spatial localization or alternatively due to the sequestering of specific factors within the telomere hyperclusters.

The extension of Sir3-bound domains upon SIR3 overexpression systematically repressed underlying transcripts genome-wide (Fig. 2D, and Fig. 2C for Tel6R) providing a parallel assessment of the validity of binding events measured by ChIP-chip. Repression was largely independent of initial transcript level (Fig. 2D) and of coding status (e.g., the right subtelomere of Chromosome VI) (Fig. 2C; Supplemental Fig. S2B). These extended silent domains (ESDs) included 100 genes that were not bound by Sir3 in WT cells. The logarithm of transcriptional repression was linearly proportional $\left(R^{2}=0.71\right)$ to the Sir3 binding signal, reflecting the absence of silencing escapers (Fig. 2D). Analysis of reads mapping to multiple loci indicated that entire gene families, characteristic of subtelomeres and $\mathrm{Y}^{\prime}$ elements, were on average repressed upon SIR3 overexpression (Supplemental Fig. S2C,E), suggesting that the subtelomeric regions devoid of chip probes are collectively silenced within ESDs.

Most genes within ESDs are not highly transcribed in WT cells, suggesting that Sir3 spreading might be limited by transcription. However, highly expressed genes like IRC7 (Fig. 2C) and DLD3 (Fig. 3B) were not excluded from ESDs and were repressed upon SIR3 overexpression. Both genes belong to the top $10 \%$ of most expressed genes and to the top $20 \%$ of most frequently transcribed genes in wild-type cells (Pelechano et al. 2010). Similarly, at seven subtelomeres at least one gene within the ESD had higher read density than the gene adjacent to the ESD (Fig. 2E). Furthermore, genes found immediately before and after the end of ESDs showed comparable transcript levels (Fig. 2F). Therefore, transcriptional activity per se did not appear sufficient to limit Sir3 spreading when Sir3 is overabundant.

The limitation of Sir3 spreading could be the consequence of the counterselection of cells silencing essential genes because ESDs did not contain any and three ESDs ended right before three essential genes. However, we do not favor this hypothesis for two main 
A
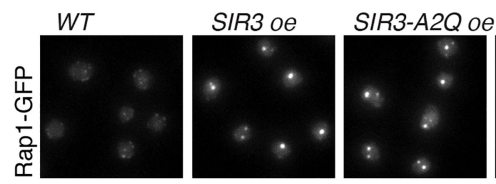

B

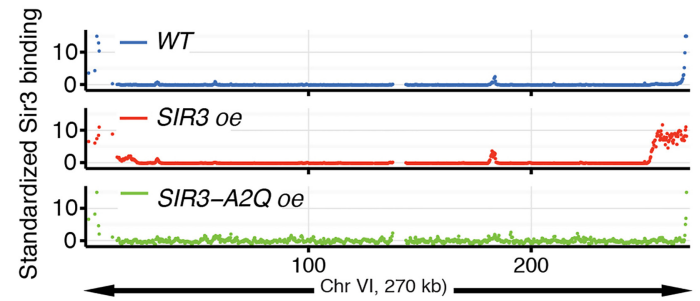

$\frac{\bar{c}}{\mathrm{c}}$
$\frac{1}{\mathrm{c}}$
$\frac{1}{\mathrm{c}}$

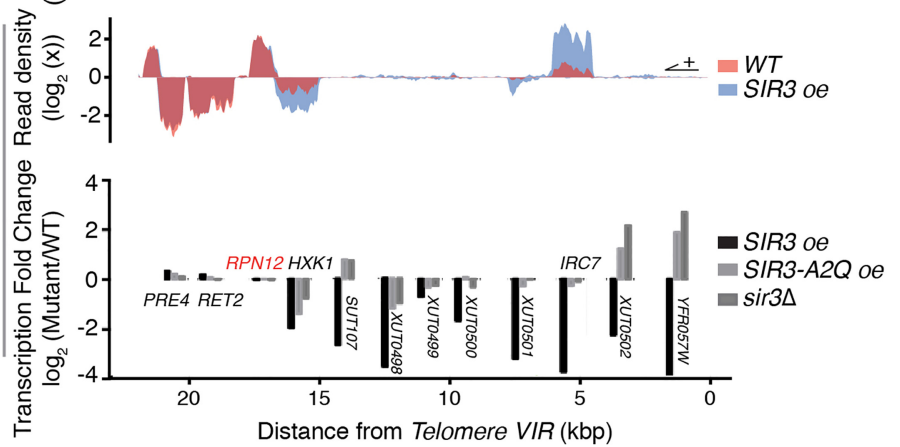

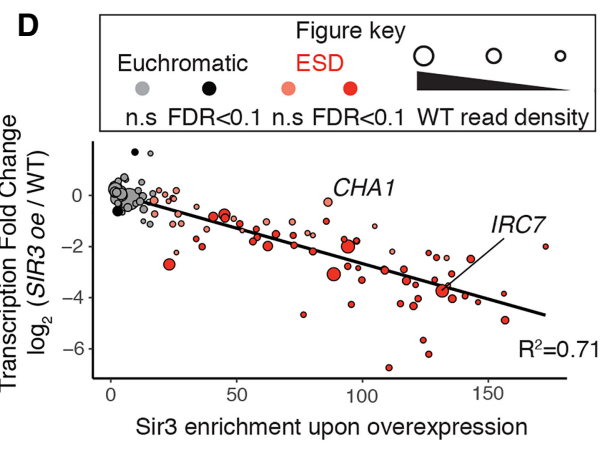

E

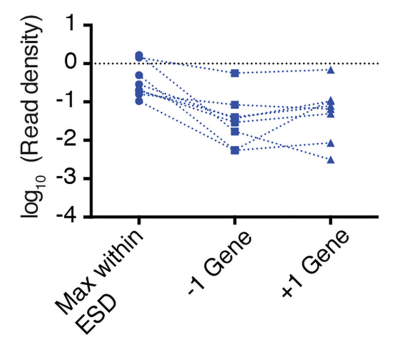

$\mathbf{F}$

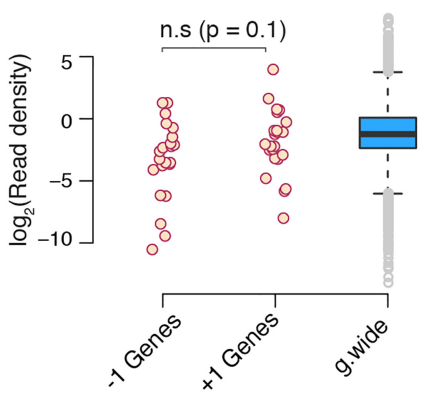

Figure 2. Sir3 extended domains are silenced and restricted to subtelomeres. (A) Representative Rap1-GFP images of exponentially growing strains with different Sir3 amount or expressing the SIR3-A2Q point mutant allele. (B) Chromosome-wide binding of Sir3 in the same strains as in $A$ and blow-up on subtelomere VIR. Enrichment is shown as the standardized IP over input and scale is thresholded at 15 for visualization purposes. (C) Total RNA-seq read density and corresponding transcriptional fold change along subtelomere VIR in indicated exponentially growing (OD600 nm 1) strains. (D) Sir3 binding and corresponding transcription changes of subtelomeric genes (distance from chromosome end $<50 \mathrm{~kb}$ ) upon overexpression of SIR3. Genes showing infinite fold change values were excluded from this plot. Color code indicates if a gene is annotated as within ESDs (see Methods) and shade indicates significance $(F D R<0.1)$ of the detected changes. Read density in WT cells is proportional to the disk area. Black line corresponds to linear fitting of the data, and corresponding $R^{2}$ value is shown. (E) Exemplification of the seven subtelomeres at which a gene within the ESD shows larger transcript amount than the genes located at the end of the domain. $(F)$ Read density of genes located before and after the end of extended silent domains compared to genome-wide distribution, statistical test: Wilcoxon test, paired values.

A
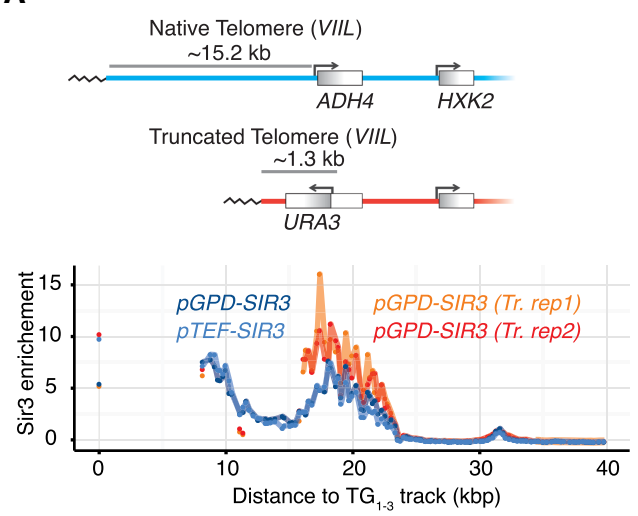

B

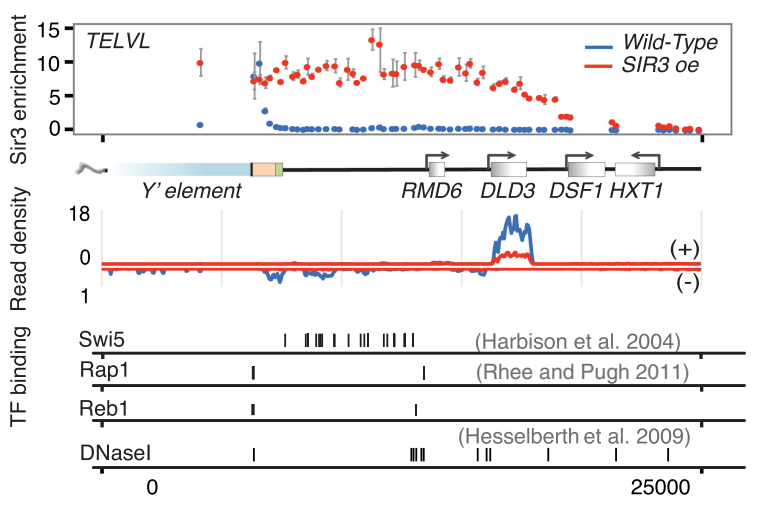

Figure 3. End of extended silent domains is defined locally and independently of transcriptional activity. (A) Sir3 binding at native and truncated TELVIIL, $x$-Coordinates correspond with the native telomere TELVIIL. (B) Sir3 binding at TELVL in WT and Sir3-overexpressing ( $p G P D$-SIR3) strains. Transcription factor (TF) binding and DNase I hypersensitive sites along TELVL are shown. 
reasons. First, we did not detect significant decreases in mRNA levels for these genes upon Sir3 overexpression. Second, none of these genes show any haploinsufficiency phenotype (Deutschbauer et al. 2005), arguing that a weak repression would likely not be counterselected. We thus conclude that extended Sir3 spreading led to efficient gene silencing of the underlying genes, and gene activity did not account for the end of ESDs.

\section{ESDs are not limited by distance from the telomere or by barrier TF elements}

To test whether the distance from the telomere limits Sir3 spreading, we compared Sir3 spreading at a WT telomere VIIL against a $15-\mathrm{kb}$ truncated version (Fig. 3A). In both cases, Sir3 binding ended within the HXK2 promoter, with a somewhat sharper decline rate in the truncated strain. This suggests that the Sir3 spreading boundary is either defined relative to the chromosome core or is a local feature. Focusing on silent domain ends, we quantified the slope of Sir3 binding at each subtelomere in the strains overexpressing SIR3, when sufficient data were available (24/32 subtelomeres). We found that the slope at the end of a silent domain did not correlate with the distance from the telomere (i.e., nucleation point), and there was no correlation with the groups defined based on sensitivity to Sir3 dosage (Supplemental Fig. S3A). Thus, when Sir3 is in excess, the delineation of the ESD did not depend on the distance from the nucleation site. To investigate whether DNA sequence-specific barrier elements confine Sir3 ESDs within subtelomeres, we evaluated the available binding data of 10 transcription factors (TF) with proposed barrier activity: Adr1, Gcn4, Rgt1, Hsf1, Sfp1, Reb1, Abf1, Leu3, Swi5 (Harbison et al. 2004); Rap1 (Rhee and Pugh 2012); and Tbf1 (Preti et al. 2010). At 12 subtelomeres, we identified bound TF sites at genes corresponding to the ESD limit (Supplemental Fig. S3C). However, each of these TFs was also bound elsewhere within the ESD (Fig. 3B), indicating that they were not sufficient to limit the spreading of Sir3. Only the three subtelomeres categorized as insensitive to Sir3 levels (group 4) contained known barrier elements flanking Sir3-bound domains: a $t R N A$ gene at subtelomere $I I L$, a previously identified barrier sequence homologous to the left barrier of $H M L$ (Bi 2002) at the subtelomere $X I R$, and the I silencer at subtelomere IIIL (Supplemental Fig. S3B). Thus, in most cases, none of the previously identified barrier elements that we could probe was sufficient to block Sir3 spreading.

\section{Sir2 does not limit the majority of extended silent domains}

We considered that Sir3 spreading might be limited by the capacity of Sir2 to deacetylate H4K16. We first monitored the genomewide occupancy of Sir3 in strains overexpressing Sir2. We found that Sir 2 overexpression had a weaker impact than $9 \times$ Sir 3 overexpression (Supplemental Fig. S4A). Sir3 spreading in cells cooverexpressing Sir2 and Sir3, or overexpressing Sir3 alone were identical at most subtelomeres $(19 / 26)$, as illustrated by their mean ChIP profile (Fig. 4A). In the remaining seven cases, Sir3 spreading was increased by co-overexpression of Sir2, slightly extending the average profile of Sir3 binding (Supplemental Fig. S4B). It is noteworthy that the further extended silent domains remained devoid of essential or tRNA genes. Thus, Sir2 activity did not generally limit the spread of heterochromatin, even when Sir3 is in excess.

\section{ESDs encompass known domains of Sir3 extension}

We compared how Sir3-bound domains extend upon overexpression to known situations of Sir3 binding extension: Sir3 spreading in H3 tail mutants (Sperling and Grunstein 2009) and in cells blocked in G1 by alpha-factor treatment (Mitsumori et al. 2016). As shown in Figure 4, ESDs encompass the domains bound by Sir3 in H3 tail mutants or in G1-blocked cells. Although in the H3 tail mutant, Sir3-bound domains increased only at half subtelomeres, in these cases Sir3 binding profiles were very similar to those observed upon SIR3 overexpression (Fig. 4B; Supplemental Fig. S4B). In contrast, Sir3 binding in G1-blocked cells appeared to cover domains identical to ESDs but with a binding signature qualitatively different, as only a low magnitude binding signal is observed in the extended Sir3 binding domains.

Together, this shows that Sir3-bound domains in G1-blocked cells or in H3A4-30 are contained within ESDs, although Sir3 is not overexpressed in these conditions. This suggests that the same determinants of Sir3 restriction are at play in all these contexts. Finally, the similarities of Sir3-bound domains in those conditions may indicate that ESDs correspond to chromosomal features that exist independently of Sir3 dosage.

\section{ESDs coincide with a preexisting chromatin landscape}

To identify potential chromatin determinants of ESDs, we analyzed the genome-wide distribution of 27 histone marks or variants
A Sir3 average binding profile (HM excluded)

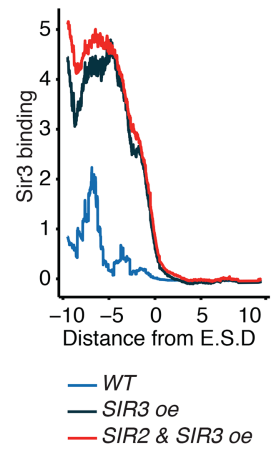

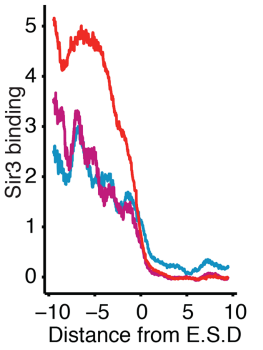

- H3 $44-30$ (A.S.) - G1 block (M.R.) — SIR2 \& SIR3 oe

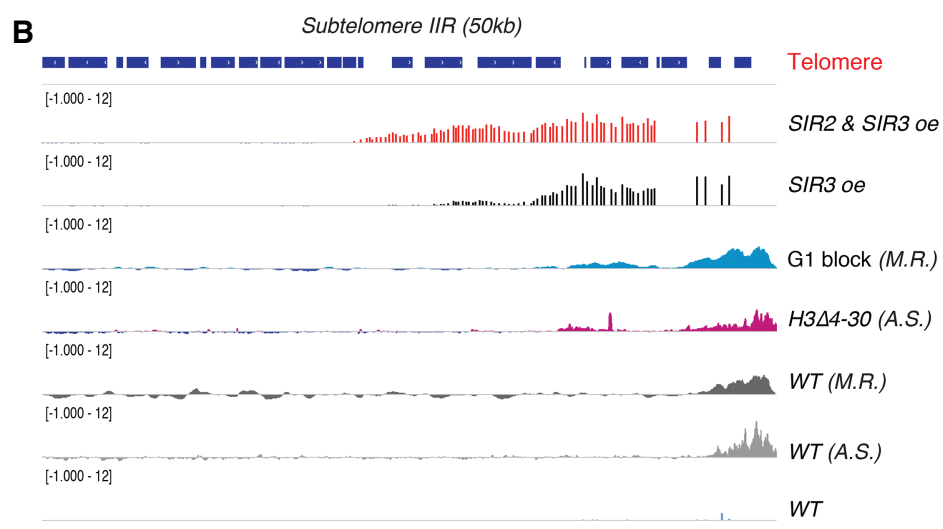

Figure 4. ESDs encompass known domains of Sir3 extension. ( $A$ ) Moving average of Sir3 binding (block=1000 bp, window $=10$ ) at the end of ESDs in the indicated conditions or mutants. $(B)$ Genome browser visualization of Sir3 binding at subtelomere IIR; all data are shown as Z-score with a lower bound of -1 and an upper bound of 12 . 
(Schulze et al. 2009; Weiner et al. 2015). We first computed the correlation between Sir3 binding signals and histone modifications across the first centromere proximal 50-kb flanking X-core elements (Fig. 5A). Consistent with previous results, we observed the expected anti-correlation between Sir3 binding and H4K16 acetylation in WT cells with a Pearson's correlation coefficient of -0.45 (Fig. 5A, and exemplified in Fig. 5B). Correlation between Sir3-A2Q binding and histone marks ranged from -0.25 to 0.2 , providing a negative control. Generally, in wild-type cells Sir3-bound nucleosomes were depleted of most histone marks, with the exception of H4R3 methylation and H2A phosphorylation (H2AP), which were enriched within silent domains (Supplemental Fig. S5A), as reported earlier (Yu et al. 2006; Szilard et al. 2010). We observed that Sir3 binding signal was better correlated with several histone marks in all conditions corresponding to extended binding of Sir3 (H3 tail mutants, G1-blocked cells, SIR3 overexpression, and SIR2 and SIR3 co-overexpression) than in asynchronous wild-type cells. Namely, Sir3 binding signal correlated better with histone $\mathrm{H} 3$ methylation and histone H2A phosphorylation (Fig. 5A,B), the highest correlation values being with Sir3 binding signal in cells co-overexpressing SIR2 and SIR3 (Pearson's correlation coefficient of -0.49 with $\mathrm{H} 3 \mathrm{~K} 79 \mathrm{me} 3$ and 0.72 with H2AP). Although Sir3 binding in G1-blocked cells remained negatively correlated with H4K16 acetylation status, this anti-correlation was much weaker in $\mathrm{H} 3$ tail mutants and even lower in strains overexpressing SIR3. This suggests that H4K16 acetylation might limit Sir3 binding in G1-blocked cells but not in H3 tail mutants or upon SIR3 overexpression.

To investigate a potential link between chromatin and the consequences of SIR3 overexpression, we compared histone marks enrichment at the flanks of wild-type silent domains among the fragile, progressive, and robust subtelomere groups defined in Figure 1. We observed that some histone marks are on average differentially enriched in the three groups (Supplemental Fig. S5A). The most pronounced differences (Kruskal-Wallis, $P$-value $<$ $10^{-4}$ ) concerned H3K79me2 and the acetylation of the H4 tail lysines $(\mathrm{K} 5,8,12$ but not K16: ANOVA, $P$-value $=0.55)$ that showed higher levels within ESDs of progressive telomeres than at fragile telomeres and even higher levels at robust telomeres.

Because acetylation of $\mathrm{H} 4 \mathrm{~K} 5,8,12$ reduces Sir3 affinity for $\mathrm{H} 4$ in a cumulative manner (Carmen et al. 2002), those differences could contribute to the differential spreading observed between those groups of subtelomeres upon overexpression of SIR3. Similarly, Sir3 H3K79me2 could limit Sir3 spreading at progressive and robust telomeres when Sir3 is overexpressed ninefold but not above.

H3K79me, and the histone variant H2A.Z, previously reported as antagonistic to SIR spreading, showed low levels in wild-type

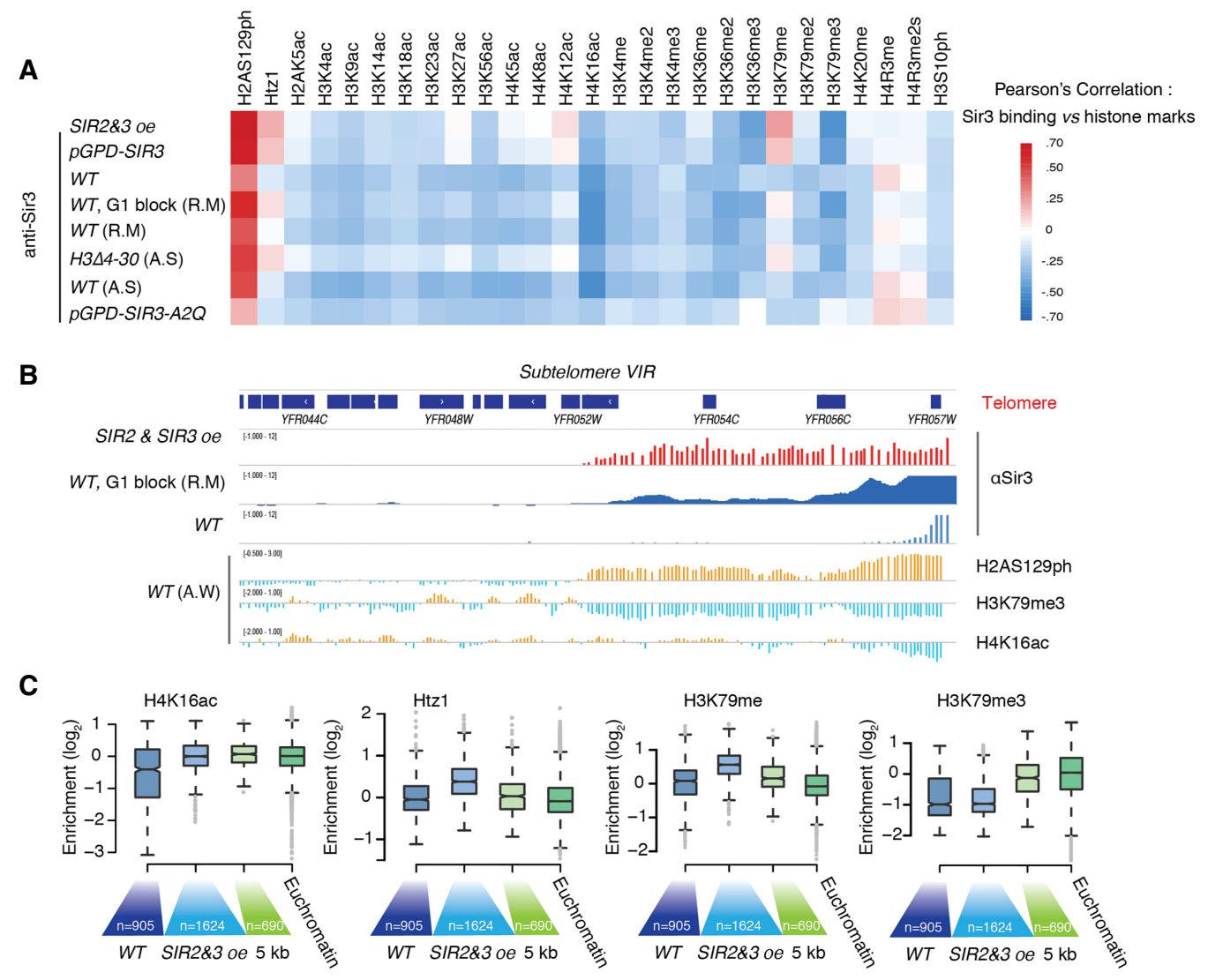

Figure 5. Extension of silent domains predicts major subtelomeric chromatin transitions. ( $A$ ) Pearson's correlation matrix between Sir 3 binding and histone marks, SIR3 oe corresponds to yAT1254 and SIR2 and SIR3 oe to yAT1668. Histone modification data from Weiner et al. (2015) for all marks except H3K79me2 (Schulze et al. 2009). (B) Genome browser visualization of Sir3 binding in WT, pGPD-SIR2 pGPD-SIR3 strains, in G1-blocked cells, in H3 44-30 mutants and selected histone modifications (from Weiner et al. 2015) in WT strains at TELVIR. (C) Distribution of selected histone marks relative to H3 (data from Weiner et al. 2015) along wild-type silenced domains and within the contiguous subtelomeric domains accessible to Sir3 upon overexpression. For comparison, the distributions of those marks within the $5 \mathrm{~kb}$ contiguous to the end of extended silent domains as well as within euchromatin (i.e., ESD excluded, $n=49,313$ ) are shown.

\section{Genome Research}

www.genome.org 
silent domains similar to the bulk of the genome but were enriched within the ESDs and returned to background levels past ESDs (Fig. $5 \mathrm{~A}, \mathrm{C})$. This suggested that these chromatin features did not efficiently block Sir3 spreading when Sir3 is overabundant or in G1 phase.

In contrast, $\mathrm{H} 3 \mathrm{~K} 4 \mathrm{me} 3, \mathrm{H} 3 \mathrm{~K} 36 \mathrm{me} 3$, and $\mathrm{H} 3 \mathrm{~K} 79 \mathrm{me} 3$ were only present after the ESD terminus (Fig. 5C; Supplemental Fig. S5B). We reasoned that the longer intergenes present within subtelomeres might bias our analysis, artificially leading to the depletion of marks associated to gene bodies. To control for this potential artifact, we separated promoter nucleosomes $(-3,-2,-1)$ from gene body nucleosomes and obtained essentially the same results (Supplemental Fig. S5C).

Thus, the subtelomeric chromatin landscape exhibits more similarities with Sir3 binding when it is extended than with a Sir3-bound region in a wild-type asynchronous population.

In a complementary approach, we focused on Sir3 binding signal at the ends of ESDs. We classified each subtelomere according to Sir3 binding signal's area under the curve (AUC), computed on the logistic-like fit of the Sir3 binding signal in ESDs (Methods). At the 10 telomeres showing the highest AUC, some histone marks displayed sharp transitions, particularly H3K79me3 and H2AP (Fig. 6A). In contrast, the 10 subtelomeres with the lowest Sir3 binding AUC at ESD ends showed rather smooth changes both for Sir3 binding and for these marks (Fig. 6A; Supplemental Fig. S6A). Thus, ESDs correlated with a preexisting chromatin landscape defined by specific histone modifications, low levels of H3K79me3 and H3K36me3, and high levels of H2AP.

\section{The methyltransferase Dotl is essential for viability when SIR3 is overexpressed}

As H3K79 methylation has been shown to impair the binding of Sir3 to histone peptides and to nucleosome in vitro (Altaf et al. 2007; Wang et al. 2013), it appeared as a good candidate to limit ESDs. To test this hypothesis, we overexpressed SIR3 in this absence of Dot1, the only methyltransferase responsible for H3K79 mono-, di-, and trimethylation. We found that the dot1A pGPD-SIR3 strains were sick and generated suppressors upon streaking. To avoid artifacts due to these potential escapers, we selected dot1 $1 \triangle$ PPD-SIR3 clones in the presence of $5 \mathrm{mM}$ nicotinamide (NAM), which inhibits Sir2 activity and thus silencing (Bitterman et al. 2002). After the selection of positive clones, we assessed the growth of these mutants on medium without NAM, allowing silencing to initiate (Osborne et al. 2009). Above ninefold overexpression of SIR3, dot14 mutants exhibited growth defects that were proportional to Sir3 amounts and rescued by Sir2 inhibition (Fig. 6B). In contrast, the H3K4 and H3K36 methyltransferases, Set 1 and Set2, and the histone deacetylase, Rpd3, were all dispensable to maintain cell growth in the presence of high Sir3 dosage (Supplemental Fig. S6B). A dot1A strain overexpressing the spreading-defective SIR3-A2Q point mutant was viable, further supporting that the requirement for Dot 1 is to restrict the spread of Sir3 and not the clustering of telomeres (Supplemental Fig. S6B). Furthermore, co-overexpression of DOT1 and SIR3 led to loss of silencing, showing that H3K79 methylation prevails on Sir3 binding even when SIR3 is overexpressed (Supplemental Fig. S6C).

\section{H3K79 methylation protects euchromatin from the spread of silencing}

To identify $\mathrm{H} 3$ and $\mathrm{H} 4$ histone residues involved in the limitation of Sir3 spreading, we set a genetic screen based on the Synthetic
Gene Array (Dai and Boeke 2012). H3K79R was the sole histone point mutant having growth defects that could be rescued by 5 mM NAM treatment (Fig. 6C; Supplemental Fig. S6D), indicating that H3K79 methylation plays a key role in limiting Sir3 spreading. In contrast, the $H 4 K 16 R$ mutant had nonsignificant growth defects (Fig. 6C; Supplemental Fig. S6E), consistent with a rather subtle influence of H4K16 on the maximal extent of silencing upon SIR3 overexpression.

Next, we observed Sir3 binding events that led to cell lethality in DOT1 deleted mutants overexpressing SIR3. To do so, cells were first grown in the presence of NAM and then released into fresh medium for $8 \mathrm{~h}$. Sir3 binding in cells overexpressing SIR3 released from NAM was very similar to what we observed in cells grown in the absence of NAM (Pearson's correlation coefficient, 0.9). In contrast, in dot1 1 mutants overexpressing SIR3, Sir3 spread beyond ESDs at several subtelomeres and bound numerous euchromatic sites (Fig. 6D,E). The subtelomeric extension of Sir3 spreading encompassed four essential genes as shown for subtelomeres 3L, 3R, and 2L (Fig. 6D; Supplemental Fig. S6E) likely accounting for the lethality of this strain. In addition, Sir3 overcame the three previously identified barriers, including the tRNA gene present at the border of subtelomere 2L that was insensitive to Sir2 and Sir3 overexpression in otherwise wild-type cells (Supplemental Fig. S6E).

To define the inhibitory effect of each H3K79 methylation state on Sir3 binding, we compared Sir3 binding in dot1 mutants overexpressing SIR3 to the levels of mono-, di-, and trimethylation of H3K79 deposited by Dot1 in WT cells. We observed that in the absence of Dot1, Sir3 was binding loci that were enriched for H3K79 trimethylation but depleted for H3K79 mono- and dimethylation in wild-type cells (Fig. 6F). This suggests that it was the trimethyl state of H3K79 that inhibited Sir3 binding and so prevented heterochromatin formation within euchromatin.

Accordingly, SIR3 overexpression was also lethal in rad64 cells (Supplemental Fig. S6F); these lack H3K4me3 and H3K79me3 but not H3K79me1 and 2 (Ng et al. 2002; Schulze et al. 2009). We thus conclude that H3K79me1 and me 2 could contribute to but are not sufficient for blocking Sir3 binding within euchromatin.

\section{ESDs coincide with discrete domains that segregate subtelomeric features}

We identified discrete subtelomeric domains corresponding to the maximal extension of Sir3-bound domains. We next sought to identify regulators of genes found in these domains by screening a compendium of more than 700 mutants (Kemmeren et al. 2014). We classified subtelomeric genes into four groups: (1) genes or pseudogenes associated with middle repeat elements (telomeric); (2) genes bound by Sir3 in WT cells; (3) genes bound by Sir3 only upon SIR3 overexpression; and (4) genes bound Sir3 only upon co-overexpression of SIR 2 and SIR3. Groups 3 and 4 correspond to ESDs. We also considered the group of genes located within $10 \mathrm{~kb}$ from the end of ESDs and located between 10 and $20 \mathrm{~kb}$ from ESD ends as a control.

For each mutant, we tested if the proportion of differentially expressed genes $\left(\left|\log _{2}(\mathrm{FC})\right|>2\right)$ within a subtelomeric domain was higher than expected by chance, considering the effect of each mutation on the rest of the genome. We identified genes whose mutation affects specific subtelomeric subdomains (Fig. 7A). As expected, deletion of any of the SIR had localized effects within the telomeric and WT Sir3-bound domains.

Our analysis confirmed mutants previously known to affect subtelomeric transcription. Telomerase components and the 
A
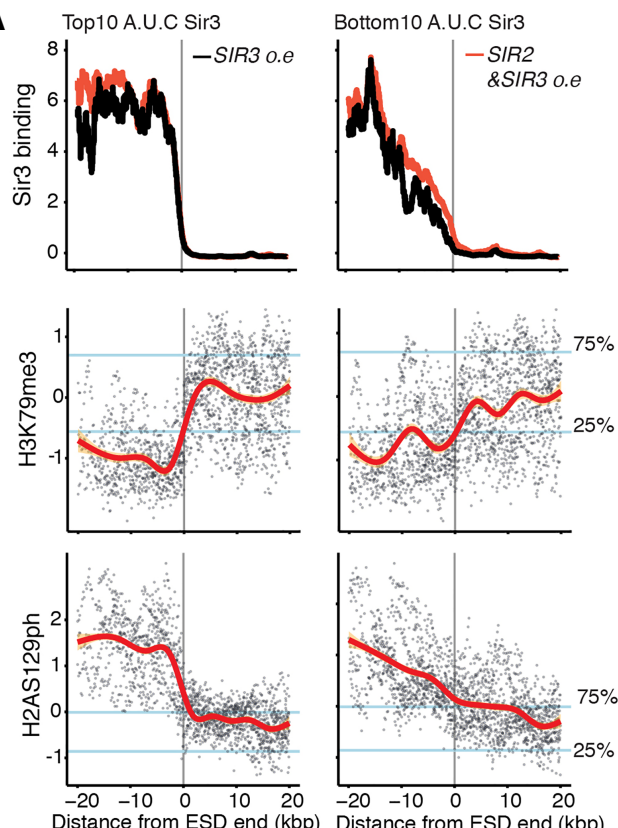

C

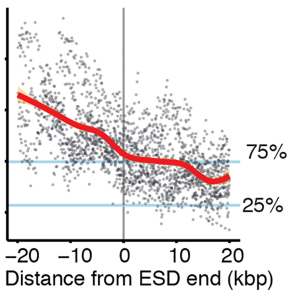

D

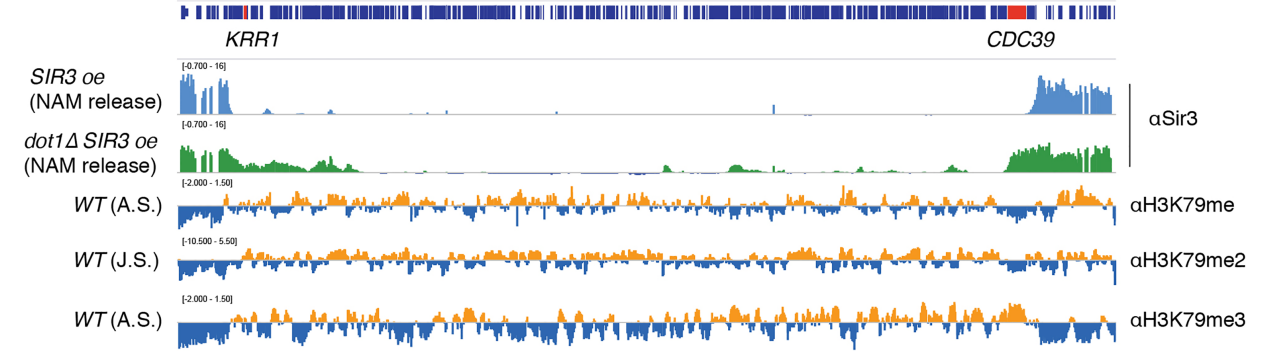

E

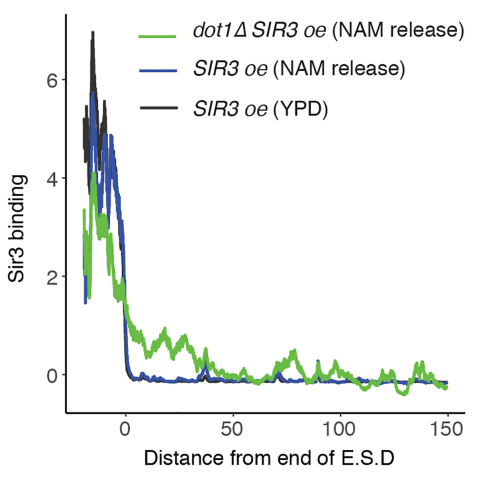

B

WT

PADH-SIR3

PTEF-SIR3

pGPD-SIR3

dot1 $\triangle$

dot1 $\triangle P A D H-S I R 3$

dot1 $\triangle$ PTEF-SIR3 dot1 $\triangle P G P D-S I R 3$

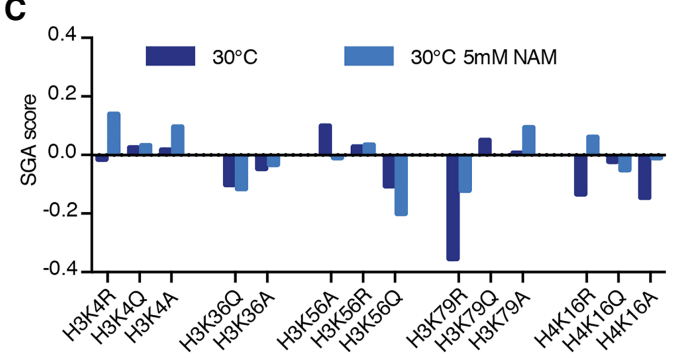

Chromosome III

F

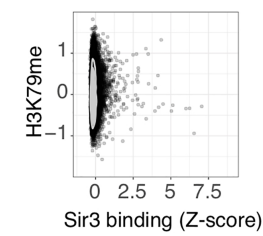

SIR3 oe (NAM release), ESD excluded
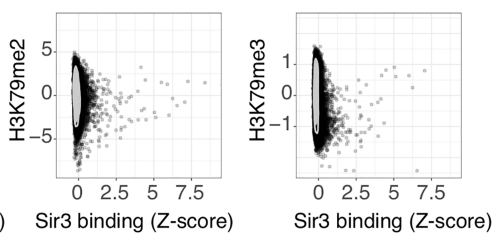

dot1 $\triangle$ SIR3 oe (NAM release), ESD excluded
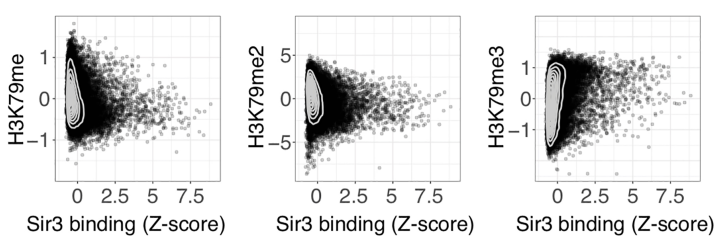

Figure 6. $\mathrm{H} 3 \mathrm{~K} 79$ methylation is key to sustaining viability upon Sir3 overexpression. (A) Moving average of Sir3 binding at telomeres (10-kb windows, 10-bp step). The top and bottom 10 telomeres with regard to Sir3 signal in strains overexpressing SIR 2 and SIR3 were plotted separately. H3K79me 3 data were obtained from Weiner et al. (2015). Blue lines indicate genome-wide lower and higher quartiles for each mark. The red line corresponds to the local smoothing of histone modification data. (B) Dilution assay to probe viability of dot1 mutants upon overexpression of SIR3. Cells were constantly grown in the presence of $5 \mathrm{mMNAM}$ prior to this assay. Cells were grown overnight, and 0.5 OD of cells were plated in $5 \times$ serial dilutions on YPD or YPD $5 \mathrm{mM}$ NAM. (C) Growth score of selected histone point mutants on galactose plates (Sir3 inducing conditions) with or without NAM compared to glucose plates (Sir3 dosage is WT). (D) Genome browser visualization of Sir3 binding in $p G P D-S I R 3$ and dot $1 \triangle P G P D$-SIR3 strains $8 \mathrm{~h}$ after being released from $5 \mathrm{mM} \mathrm{NAM.} \mathrm{KRR1}$ and CDC39, labeled in red, are essential genes. H3K79 methylation enrichment was obtained from Weiner et al. (2015) for H3K79me and H3K79me3 and from Schulze et al. (2009) for H3K79me2 (Mat score is shown). ( $E$ ) Moving average of Sir3 binding (block $=1000 \mathrm{bp}$, window $=10 \mathrm{bp}$ ) at the end of ESDs in $p G P D-S I R 3$ and dot $1 \triangle p G P D$-SIR3 strains $8 \mathrm{~h}$ after being released from $5 \mathrm{mM}$ NAM. $(F)$ Dot plot showing Sir3 enrichment in the indicated strains and condition against the enrichment for each methylation level of $\mathrm{H} 3 \mathrm{~K} 79$. $\mathrm{H} 3 \mathrm{~K} 79$ methylation data were obtained from the same source as in $D$ and averaged at each ChIP-chip probe.

\section{Genome Research}

www.genome.org 
A

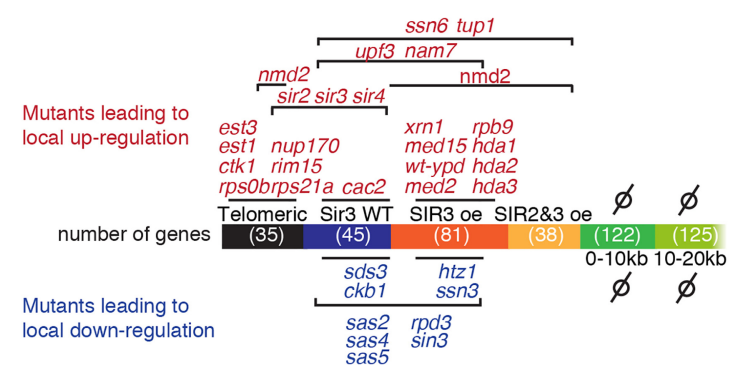

C

H3K79me3 from different view points

- ESD ends HAST ends - Synteny defined subtelomere

$\downarrow$

Linear fitting along profiles

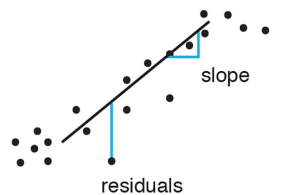

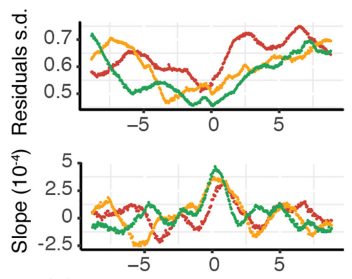

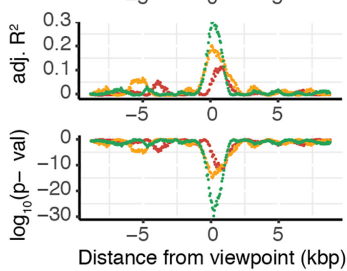

B

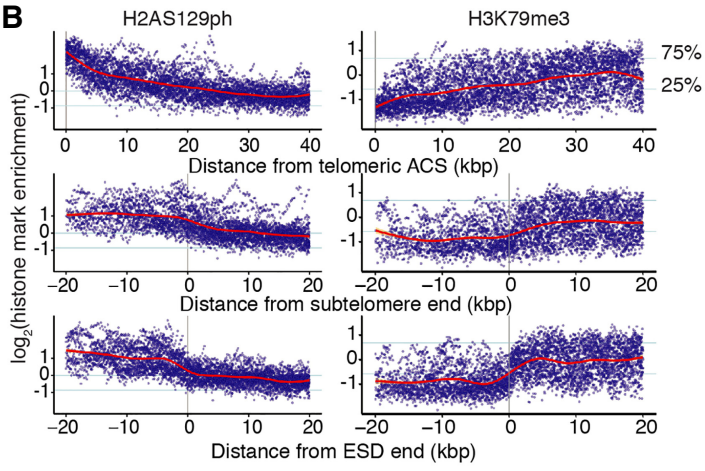

D

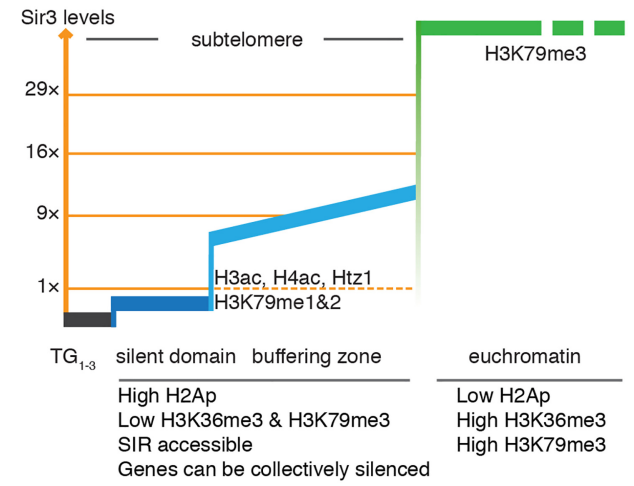

Figure 7. Extension of silent domains reveals new aspects of subtelomeric structures. $(A)$ Localized effects of mutations affecting subtelomeric transcription. The different subtelomeric subdomains are defined according to Sir3 binding. The number of genes within each domain is in parentheses. Mutant names are positioned according to the domain(s) within which the proportion of genes up- or down-regulated (log(FC) $>1$ or $<-1)$ is significantly elevated (hypergeometric law, with Bonferroni correction $n=703$ ). (B) Distribution of H3K79me3 and H2AS129ph (obtained from Weiner et al. 2015) relative to different subtelomeric viewpoints. Blue lines indicate genome-wide lower and higher quartiles for each mark. The red line corresponds to the LOESS smoothing of histone modification data. (C) Quantification of H3K79me3 transition in function of different genomic viewpoints. Shown are the results of a linear model fitting of the histone mark enrichment data (residuals standard deviation, slopes, $R^{2}$, and $P$-values) over 2.35 -kb windows every 50 bp. (D) Model depicting how extending silent domains enables to uncover consistent subtelomeric domains delimited by chromatin mark transitions.

nucleoporin NUP170 (Van De Vosse et al. 2013) up-regulates specifically the most telomeric group of genes in our analysis, whereas the mediator complex tail proteins Med2, Med15, and Gal11 (Lenstra et al. 2011; Peng and Zhou 2012), the Hda1/2/3 complex, and the general repressors Tup1/Cyc8 specifically affect genes located within ESDs (Fig. 7A). The localized enrichment for downregulated genes in $r p d 3$ or sas $2 / 4 / 5$ mutants did not extend beyond the ESDs. This enrichment for down-regulated genes was likely the consequence of increased spreading of the SIRs in those mutants. Therefore, SIR-dependent silencing in those mutants seemed not to extend beyond ESDs, further reinforcing the notion that ESDs represent the maximal extension of SIR-dependent silencing. Lastly, enrichment for down-regulated genes in the tup1 and ssn6 mutants decreased abruptly at the end of ESDs (Supplemental Fig. S7A,B) and no mutant had enriched impact on the genes in the $10 \mathrm{~kb}$ immediately adjacent to ESDs. Thus, the domains defined by the saturated expansion of silent chromatin encompassed the subtelomeric domains in which known mutants affecting subtelomeres have an effect.

Last, we probed how the ESD point of view segregates subtelomeric properties as compared to other definitions of subtelomeres: distance from telomeres, from the first essential gene, the end of HAST domains (defined as Hda1 affected subtelomeric regions) (Robyr et al. 2002), or subtelomeres based on synteny conservation across close relative species (Yue et al. 2017). ESDs often extended within the core chromosome as defined by synteny, more than $10 \mathrm{~kb}$ at five subtelomeres (Supplemental Fig. S7C), showing that ESD constituted a different definition of subtelomeres. ESD and HAST domains ended at similar location at eight subtelomeres $(<3 \mathrm{~kb})$ but HAST domains, which are only defined at 22 subtelomeres, generally extended beyond ESD (Supplemental Fig. S7D). Furthermore, the transitions of the H2AS129ph (H2AP) and H3K79me3 histone marks (Fig. 7B) are evidently sharper from the ESD end viewpoint than from the distance from X-core sequence or syntenically defined subtelomere end. Quantification of H3K79me3 transition by systematic fitting of a linear model around the transition zones confirmed that ESDs better capture H3K79me3 subtelomere to euchromatic transitions than syntenically derived subtelomere or HAST domain ends (Fig. 7C). Similar quantification for H2P transition showed than ESDs and HASTs comparably segregated H2AP although ESDs included four more subtelomeres, both viewpoints being more efficient than syntenically defined subtelomere ends (Supplemental Fig. S7E). Thus, probing the maximal extent of silencing domains revealed discrete subtelomeric domains delimited by histone mark transition zones and provides a new definition of subtelomeres (Fig. 7D).

\section{Discussion}

The Sir complex has been a model for chromatin complex propagation and gene silencing for decades. Pioneer studies demonstrated 
that increasing the dose of Sir3 extends silenced domains at subtelomeres (Renauld et al. 1993; Pryde and Louis 1999), a property common to several heterochromatin complexes. However, there has been controversy on the generality of this finding at natural telomeres (Pryde and Louis 1999; Ellahi et al. 2015), and the details of this process remain unclear. Here, we systematically studied the impact of increasing Sir2 and Sir3 dosage on the propagation of the SIR complex and on genome-wide transcription.

Gradual overexpression of Sir3 revealed that the spreading of Sir3 over subtelomeres reached saturation at Sir3 levels between $9 \times$ and $16 \times$, suggesting the existence of fixed borders. Similarly, telomere clustering increased at $9 \times$ and $16 \times$ Sir3 levels but not above. Yet, the change in chromosome organization imposed by telomere hyperclustering in the center of the nucleus (Ruault et al. 2011) had a very minor impact on gene expression per se as shown by overexpression of the silencing deficient SIR3-A2Q point mutant. Conversely, spreading of wild-type Sir3 was systematically associated with decreased transcript levels of the underlying genes. This is in contrast with the situation observed in wild-type cells where Sir3 is found at rather discrete loci close to nucleation sites and with a limited effect on gene expression as previously reported (Takahashi et al. 2011; Thurtle and Rine 2014). Thus, although Sir3 spreading and impact on gene expression appears limited in laboratory strains and under standardized growth conditions, it has the potential to spread over several kilobases, creating domains of silent chromatin that we named ESDs for extended silent domains. Unexpectedly, the response to increase in Sir3 levels differed among subtelomeres, and the concentration of Sir3 required for maximal spreading varied. Additional spreading varied from 0 to $25 \mathrm{~kb}$ in between subtelomeres, independently of middle repeat elements or chromosomal arm length and expression levels of the underlying genes. However, the domains covered by Sir3 upon overexpression shared similar chromatin marks, suggesting that the chromatin landscape dictated the extent of Sir3 spreading. Indeed, we identified H3K79me3 as the most efficient barrier to prevent heterochromatin propagation. Finally, by revealing the maximal subtelomeric domains accessible to Sir3 complex in viable cells, our work uncovered previously unknown, discrete subtelomeric domains that isolated the structural and functional features associated with subtelomeres.

\section{Different categories of Sir chromatin antagonism}

Although our data suggest the existence of fixed borders, our search for punctual barrier elements did not retrieve convincing candidates. Furthermore, native binding sites for transcription factors that block silencing when tethered to chromatin (Oki et al. 2004) were not efficient barriers to Sir3 spreading. We also observed extension of Sir3 binding within regions that were enriched with chromatin marks previously reported as antagonistic to its spreading, such as histone variant H2A.Z (Meneghini et al. 2003; Guillemette et al. 2005; Martins-Taylor et al. 2011) and monomethylated H3K79 (Altaf et al. 2007). Histone tail acetylation appears to limit spreading of Sir3 when Sir3 is present in a limiting amount. Indeed, we observed that acetylation levels of $\mathrm{H} 4 \mathrm{~K} 5,8,12$ are higher within ESDs that resist to intermediate levels of SIR3 overexpression than at other ESDs. Furthermore, H3 tail mutants show increase Sir3 spreading at some subtelomeres within ESDs (Fig. 4). Consequently, our work indicates that histone tail acetylation, H2A.Z, and specific transcription factors likely buffer the spread of the SIR rather than block it (Fig. 7D).

\section{End of extended silent domains: the specific role of Dotl}

We observed that the ends of extended silent domains coincide with major histone mark transition zones, characterized by an increased enrichment of H3K4me3, H3K36me3, and H3K79me3. Deletion of SET1 or SET2, the genes encoding the enzymes responsible for the H3K4me3 and H3K36me3, respectively, had no impact on cell growth upon Sir3 overexpression. In contrast, deletion of DOT1, which encodes the only H3K79 methyltransferase in budding yeast, was lethal in this condition. Yet viability in this strain could be rescued by inhibiting Sir2, indicating that loss of H3K79 methylation was responsible for the deadly spreading of elevated Sir3 in the absence of Dot1. Accordingly, when dot1 $\Delta$ strain overexpressing SIR3 were released from Sir2 inhibitor, Sir3 spread beyond ESDs invading four essential genes. In these conditions, Sir3 was also binding numerous euchromatic sites that were enriched for H3K79me3 (but not H3K79me1 or 2) in wild-type cells. H3K79me3 is anti-correlated with H4K5, 8, and 12 acetylation (Weiner et al. 2015), which also have the potential to limit Sir3 spreading as discussed in the preceding subsection (see also Carmen et al. 2002). This suggests that H3K79me3 was protecting nucleosomes hypoacetylated on $\mathrm{H} 4 \mathrm{~K} 5,8$, and 12 from Sir3 binding at euchromatic sites.

Because Dot1 is responsible for mono-, di-, and trimethylation of H3K79 (Stulemeijer et al. 2015) this raises the question of the relative contribution of these marks for blocking Sir3 spreading. In vitro, all three H3K79 methyl marks reduce Sir3 affinity for reconstituted nucleosomes (Martino et al. 2009; Behrouzi et al. 2016).

Here we show that upon overexpression, Sir3 spread over domains enriched for $\mathrm{H} 3 \mathrm{~K} 79 \mathrm{me} 1$ and me2, implying that these marks did not block Sir3 spreading in vivo although they slow down silencing establishment (Katan-Khaykovich and Struhl 2005; Osborne et al. 2009). In contrast, H3K79me3 signal increases abruptly where Sir3 spreading stopped. In the absence of Dot1 and upon overexpression of SIR3, we observed that Sir3 predominantly binds loci that were initially enriched for H3K79 trimethylation state. Accordingly, mutants in which H3K79me3 is abolished, but not H3H79me and H3K79me2 (Ng et al. 2002; Schulze et al. 2009), were sensitive to SIR3 overexpression. This is consistent with the observation that Sir3 associate with H3K79 mono- and dimethylation at an active subtelomeric reporter gene in vivo (Kitada et al. 2012). This is also in good agreement with crystal structure data predicting that the potential of H3K79 to form hydrogen bonds with the BAH domain of Sir3 would progressively decrease with H3K79 methylation to be abolished by H3K79me3, thereby decreasing Sir3 affinity to nucleosomes (Armache et al. 2011; Arnaudo et al. 2013).

All together our work demonstrates that H3K79 methylation, predominantly the trimethyl state, restricts silencing within subtelomeric regions thus protecting euchromatin. Because the occupancy of this mark is independent of transcription rate (Schulze et al. 2009), this offers the attractive possibility of preventing heterochromatin spreading independently of transcription.

\section{Subtelomeric specificities}

In most organisms, specific features of chromosome ends extend beyond telomeres, within domains generally referred to as subtelomeres (Louis et al. 2014). In budding yeast, several viewpoints enable the identification of diverse subtelomeric features, including a lower gene density, and a faster evolution than the core genome (Yue et al. 2017). 
The chromatin landscape also exhibits specific features within domains located proximal to chromosome ends (Robyr et al. 2002; Millar and Grunstein 2006; Matsuda et al. 2015). The first is undoubtedly the presence of heterochromatin, which has a unique signature in terms of histone marks. However, specific properties associated with chromosome ends often extend beyond heterochromatic domains (Millar and Grunstein 2006; Matsuda et al. 2015). At most $S$. cerevisiae subtelomeres, Hda1-affected subtelomeric (HAST) domains (Robyr et al. 2002) and Htz1 activated (HZAD) domains (Guillemette et al. 2005) lie contiguous to SIRsilenced chromatin. In addition, phosphorylation of H2AS129 and monomethylation of H3K79 also extend further away than SIR-silenced domains. Here we show that ESDs possess a consistent chromatin signature-namely, these domains are enriched for H2AP, Htz1, and depleted of trimethylated histone H3, which levels show sharp transition at the end of ESDs. Consistently, Htz1sensitive genes are enriched in these domains. Furthermore, considering the end of ESDs as a boundary between subtelomeres and the core genome segregates genes sensitive to the depletion of chromatin modifiers such as Hda1, Tup1/Ssn6, or Sas2 better than other definitions of subtelomeres (Supplemental Fig. S7D, E). Similarly, ESDs segregated transition of histone marks such as H3K73me3 better than other definitions of subtelomeric domains did. Thus, ESDs coincide with discrete subtelomeric domains isolating structural and functional features and could provide an alternative definition of subtelomeres. Furthermore, domains defined by ESD contain genes than can collectively be repressed in nonstressful conditions, a notion consistent with the idea that subtelomeres contain genes required for response to stressful environments (Louis et al. 2014).

At most subtelomeres that we could analyze, ESDs are broader or coincide with subtelomeres defined based on synteny conservation across related species (Supplemental Fig. S7A). We and others recently showed that chromatin states impact on efficiency and outcome of both homologous recombination and nucleotide excision repair (Batté et al. 2017; Guintini et al. 2017). This raises the question of whether the specific chromatin state associated with subtelomeric domains uncovered in this study contributes to the particular evolution of those regions.

\section{Contribution of telomere proximity to subtelomeric properties}

A central question in the biology of subtelomeres is this: To what extent are the properties of subtelomeres due to their proximity to telomeres or a mere consequence of their gene content? Several studies demonstrated that the SIR complex contributes to the localization of enzymes to subtelomeres. For example, subtelomeric localization of the Okazaki fragment processing protein Dna2 is severely reduced in sir mutants (Choe et al. 2002). In addition, the kinase Tel1 responsible for H2A phosphorylation in subtelomeric regions is present at telomeres, but H2AP levels depend mainly on the integrity of the SIR complex (Kitada et al. 2011). Regions enriched for H2AP coincide with ESDs, suggesting that either Sir3 acts remotely or binds these regions at least transiently in wild type. Accordingly, profiling of Sir3 binding in G1-arrested cells showed low levels of Sir3 binding within ESDs (Mitsumori et al. 2016). Thus, Sir3 might influence the chromatin landscape in subtelomeric regions. How the transient presence of Sir3 during the G1 phase of the cell cycle could stabilize H2A phosphorylation is unclear. One attractive possibility is that Sir3 acts by recruiting a so far unidentified factor that would remain associated to chromatin through the whole cell cycle.

\section{Conclusion}

By taking the opposite approach to depletion studies, our work describes the dose dependency of budding yeast heterochromatin. In the presence of a large excess of silencing factors, ectopic nucleation of heterochromatin remains limited and does not impact euchromatic transcription. In contrast, we observed the extension of subtelomeric silent domains and characterized their maximal extension along with the antagonistic factors that have been overcome, such as H2A.Z or H3K79me. By scanning chromatin properties associated with Sir3 maximal binding, we uncovered major subtelomeric histone mark transition zones that functionally protect euchromatin from the spread of silencing. The long-term contribution of heterochromatin to the peculiar properties of subtelomeres will require further study.

\section{Methods}

\section{Media and growth conditions}

The strains used in this study are listed in Supplemental Table S2. Yeast cells were grown on YP with $2 \%$ glucose, raffinose, or galactose. Unless noted, all the strains used in this study were grown at $30^{\circ} \mathrm{C}$ with shaking at $250 \mathrm{rpm}$.

\section{Yeast transformation protocol}

Cells were seeded in liquid medium and grown to $0.8<\mathrm{OD}_{600}<$ 1.2. Three ODs $\left(\sim 3 \times 10^{7}\right.$ yeast cells) of cells were taken and washed with $1 \times$ TEL (10 mM EDTA at $\mathrm{pH} 8,100 \mathrm{mM}$ Tris at $\mathrm{pH} 8,1 \mathrm{M}$ Lithium Acetate), then $3 \mu \mathrm{L}$ of ssDNA (Sigma, D9156-5ML), DNA template $(0.5 \mu \mathrm{L}$ if plasmid DNA, $5 \mu \mathrm{L}$ of digested plasmid or PCR product), $300 \mu \mathrm{L}$ of $1 \times \mathrm{TEL}$, and $45 \%$ PEG 4000 solution were added. The mix was incubated for $30 \mathrm{~min}$ at $30^{\circ} \mathrm{C}$ and heat shocked for $15 \mathrm{~min}$ at $42^{\circ} \mathrm{C}$. Last, cells were plated on appropriate selective medium.

\section{Rapl foci analysis}

The image analysis is performed with a slightly modified version of the dedicated tool from Guidi et al. (2015). These modifications regard the quantification of foci and aim at providing a more accurate estimation of the quantity of fluorescence held inside each focus. The Gaussian fitting approach has been replaced by a template matching framework with a bank of 100 symmetric 2D Gaussian kernels with standard deviations ranging from 0.5 to 7 pixels. The position of each template is determined as the maximum of normalized cross correlation, whereas the most suitable template for a single focus is selected by minimizing the sum of square differences between the Gaussian template and the data within a circular mask of radius twice the standard deviation. The foci are then defined as spherical objects with radii of two times the standard deviations of the matched templates. All foci that could not be fitted were considered as a cube of dimension $5 \times 5 \times 5$. Variation of the box size did not affect overall results. The foci intensity can thus be measured as the sum of the fluorescence signal inside its sphere. Furthermore, the proportion of intensity from a nucleus held inside each of its foci is also computed.

\section{Sir3-GFP quantification}

Quantification of Sir3-GFP signal was carried using microscopy. Briefly, cells were segmented on the basis of trans signal using a modified version of CellX, and the intensity of 30 Z-stacks deconvolved imaged was summed. Deconvolution was carried out using 
MetaMorph. For each cell, the intensity/pixel was measured and normalized by the WT average.

\section{Western blots}

Protein extracts were prepared from two ODs of exponentially growing cultures (OD 1) using the post-alkaline extraction method (Kushnirov 2000). For immunoblotting, we used custom-made rabbit polyclonal antibodies raised against full-length Sir3 (1:10,000 dilution) (Ruault et al. 2011).

\section{FACS}

Cell-cycle profiles were obtained on an Accury FACS machine using CYTOX as DNA staining agent and analyzed using FlowJoX.

\section{SGA screen}

Query strain was obtained by transforming strain $y A T-1949$ with pGAL-SIR3-HPH, integrated within TRP1. The query strain was crossed with the collection of histone point mutants (Dai and Boeke 2012) following the selection steps described in Tong et al. (2001), with selection media adapted to respective genotypes. Each cross was done in quadruplicate on 1536-format plates. Once double mutants were acquired, they were transferred to one of the following: double mutant selection medium (glucose), double mutant selection medium (glucose) + 5 mM NAM, double mutant selection medium (galactose), or double mutant selection medium (galactose) $+5 \mathrm{mM}$ NAM. All strains were grown at $30^{\circ} \mathrm{C}$ and imaged after $2 \mathrm{~d}$. Image analysis and scoring were done with SGAtools (Wagih et al. 2013), where mutants growing on glucose media served as controls. Only significant changes were considered $(P$-value $<0.001)$, and a last significance threshold was chosen to only keep those mutants with absolute value scores $>0.2$.

\section{Dilution assays}

Cells were grown overnight in YPD $+5 \mathrm{mM}$ NAM before dilution. Fivefold serial dilutions are shown. Plates were grown for $2-3 \mathrm{~d}$ at $30^{\circ} \mathrm{C}$.

\section{Pellet preparation for ChIP}

A total of $20 \mathrm{OD}$ equivalent of exponentially growing cells were fixed in $20 \mathrm{~mL}$ with $0.9 \%$ formaldehyde for $15 \mathrm{~min}$ at $30^{\circ} \mathrm{C}$, quenched with $0.125 \mathrm{M}$ glycine, and washed twice in cold $1 \times \mathrm{TBS}$ pH 7.6. Pellets were suspended in $1 \mathrm{~mL} 1 \times \mathrm{TBS}$, centrifuged, and frozen in liquid nitrogen for $-80^{\circ} \mathrm{C}$ storage. For NAM release experiments, cells were grown overnight in YPD + $5 \mathrm{mM}$ NAM before dilution at 0.2 OD in YPD and allowed to grow for $8 \mathrm{~h}$ (OD 1.5).

\section{Chromatin immunoprecipitation}

All the following steps were done at $4^{\circ} \mathrm{C}$ unless indicated. Pellets were resuspended in $500 \mu \mathrm{L}$ of lysis buffer (0.01\% SDS, $1.1 \%$

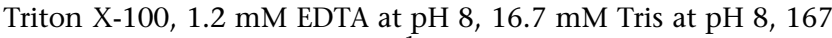
$\mathrm{mM} \mathrm{NaCl}, 0.5 \% \mathrm{BSA}, 0.02 \mathrm{~g} \mathrm{~L}^{-1}$ tRNA, and $2.5 \mu \mathrm{L}$ of protease inhibitor [SIGMA P1860]) and mechanically lysed by three cycles of $30 \mathrm{sec}$, intensity $6 \mathrm{msec}^{-1}$ with $500 \mu \mathrm{m}$ zirconium/silica beads (Biospec Products) using a FastPrep instrument (MP Biomedicals). Each bead beating cycle was followed by 5 min incubation on ice. The chromatin was fragmented to a mean size of $500 \mathrm{bp}$ by sonication in the Bioruptor XL (Diagenode) for $14 \mathrm{~min}$ at high power with $30 \mathrm{sec}$ on/30 sec off and centrifuged $5 \mathrm{~min}$ at 13,000 rpm. Ten microliters were kept to be used as input DNA. Cleared lysate was incubated overnight with $1 \mu \mathrm{L}$ of polyclonal antibody anti-
Sir3 (Ruault et al. 2011). Fifty microliters of protein A magnetic beads (NEB, S1425S) were added to the mixture and incubated for $4 \mathrm{~h}$ at $4^{\circ} \mathrm{C}$. Magnetic beads were washed sequentially with lysis buffer, twice with RIPA buffer (0.1\% SDS, $10 \mathrm{mM}$ Tris at pH 7.6, 1 mM EDTA at $\mathrm{pH} 8,0.1 \%$ sodium deoxycholate, and $1 \%$ Triton X$100)$, twice with RIPA buffer supplemented with $300 \mathrm{mM} \mathrm{NaCl}$, twice in LiCl buffer $(250 \mathrm{mM} \mathrm{LiCl}, 0.5 \% \mathrm{NP}-40,0.5 \%$ sodium deoxycholate), with TE $0.2 \%$ Triton X-100 and with TE. Input was diluted $10 \times$ with elution buffer (50 mM Tris, $10 \mathrm{mM}$ EDTA at pH 8, 1\% SDS) and beads were resuspended in $100 \mu \mathrm{L}$ elution buffer. A crosslinking reversal was performed by heating samples overnight at $65^{\circ} \mathrm{C}$. Proteins were digested with proteinase $\mathrm{K}$ in the presence of glycogen, and the remaining DNA was purified on QIAquick PCR purification columns. Finally, samples were treated with RNase A 30 min at $37^{\circ} \mathrm{C}$.

\section{ChIP-chip preparation and hybridization}

Samples used for ChIP-chip have all been analyzed by qPCR prior to microarray hybridization. For microarray hybridization, $4 / 5$ of the immunoprecipitated DNA and of the DNA from the input were ethanol precipitated and resuspended in $10 \mu \mathrm{L}$ of water (Gibco). Purified material was amplified, incorporating aminoallyl-dUTP and used as described in Guidi et al. (2015). The size of amplified fragments ( $\sim 500 \mathrm{bp}$ ) was assessed by gel electrophoresis. For each sample, $1.5 \mu \mathrm{g}$ of amplified DNA was coupled either with Cy5 (immunoprecipitated sample) or Cy3 (input sample) and hybridized on $44 \mathrm{~K}$ yeast whole-genome tiling array (Agilent) as described in Guidi et al. (2015).

\section{Microarray data acquisition, analysis, and visualization}

Microarray was imaged using an Agilent DNA microarray scanner and quantified using GenePix Pro6.1 as described in Guidi et al. (2015).

\section{Genome-wide data analysis}

Unless mentioned otherwise, data analysis was carried out using R (R Core Team 2016). All data sets were lifted over to SacCer3 when required. Histone marks data were obtained from Weiner et al. (2015), Sir3 binding in H3 tail mutants from Sperling and Grunstein (2009), Rap1 and Reb1 binding from Rhee and Pugh (2011), DNaseI hypersensitive sites from Hesselberth et al. (2009), and nucleosome turnover from Dion et al. (2007). Transcriptome data were downloaded from the website supporting the publication (Kemmeren et al. 2014). Subtelomere definition was obtained from Yue et al. (2017). Z-scores were computed using the R scale function. Criterion for the clustering of subtelomeres shown in Figure 1 were the following: First, the spreading end point was computed as the most subtelomeric probes with Sir3 binding $Z$-score $>1$ flanked by more than five probes with $Z$-score $<1$. We then applied the following criteria: "Fragile" subtelomeres: $\mathrm{d}(T E F-A D H)<2 \mathrm{~kb}<\mathrm{d}$ $(A D H-W T)$; "Unextendable": $\mathrm{d}(T E F-A D H), \mathrm{d}(A D H-W T)<2 \mathrm{~kb}$; "Robust": $\mathrm{d}(A D H-W T)<2 \mathrm{~kb}<\mathrm{d}(T E F-A D H)$; "Progressive": $\mathrm{d}(T E F-A D H)$, $\mathrm{d}(A D H-W T)>2 \mathrm{~kb}$. Of note, two subtelomeres (VIIR, XVIR) for which classification was too sensitive to a given threshold were manually curated. Euchromatic binding sites shown as Supplemental Table S1 were computed as sites away from ESDs (or present at more than $50 \mathrm{~kb}$ from a telomere when ESD are not defined) at which at least two neighboring probes are bound ( $Z$-score $>0.5$ ) by Sir3 upon overexpression of SIR3 (computed on the signal obtained from the W303 strain yAT1254).

Down-sampling of data from Sperling and Grunstein (2009), Weiner et al. (2015), and Mitsumori et al. (2016) data to the $44 \mathrm{~K}$ microarray probes for Figures $4 \mathrm{~B}$ and $5 \mathrm{~A}$ was done using $\mathrm{R}$, and

\section{Genome Research}

www.genome.org 
visual inspection of the data confirmed that down-sampling was completed without errors. Specifically, all signals located mid-distance of the previous and the next probe were averaged and allocated to the central probe. Average telomeric profiles were done by computing the mean of the signal over $10-\mathrm{kb}$ windows separated by $10 \mathrm{bp}$. The limits of extended silent domains were computed as the first probes possessing five neighboring probes that have $Z$-score inferior to 1 , starting from the telomere. Fitting of Sir3 binding data was done using MATLAB fitting toolbox using the Bisquare robustess option. The function used is $f(x)=K /\left(1+\exp \left(-r\left(-x+t_{0}\right)\right)\right)+1$, with the following fitting parameters for $K, r$, and $t_{0}$ : lower bounds: [10 0.0001 1000], Starting point: [10 0.0001 1000], upper bounds: [200 $0.0140,000]$. Area under the curve was exactly computed on the fitted signal of Sir3 binding in strains overexpressing SIR2 and SIR3, $10 \mathrm{~kb}$ before the end of silent domains and $5 \mathrm{~kb}$ after.

Mutants showing localized effects were identified by using the hypergeometric distribution, function phyper with Bonferroni correction for multiple testing $(n=703$ being the number of mutants studied showing significant transcription changes).

Linear model fitting of histone mark transition zones: For this plot, subtelomeres differing between $W 303$ and $B Y$ background were excluded $(n=3, T E L I R, T E L V I I L, T E L X I V R)$. For each viewpoint, all subtelomeres in which the viewpoint is defined were considered. First, the length on which fitting was applied was optimized as the length giving the highest $R^{2}$ around ESDs. Window sizes ranging from 1 to $5 \mathrm{~kb}$ (100 bp step) were tried for each histone mark probed. Fitting of data on windows of the defined size $20 \mathrm{~kb}$ around viewpoints with $50 \mathrm{bp}$ steps was carried with the $\mathrm{R} \operatorname{lm}()$ function.

\section{RNA-seq}

Total RNA from a 25-mL culture of exponentially growing yeasts was extracted using phenol-chloroform. Banks were constructed using the kit SOLiD Total RNA-seq, with minor modifications: RNA are zinc fragmented, and fragments with sizes ranging from 100 to $200 \mathrm{nt}$ were selected by gel purification. After reverse transcription, only fragments of size $>150 \mathrm{nt}$ are kept. Paired end $(50+35)$ sequencing was done by the Institut Curie platform. Differential expression was called using edgeR (Robinson et al. 2010; https://bioconductor.org/packages/release/bioc/html/edgeR. html), with a false discovery rate lower than 0.1 .

\section{Data access}

ChIP-chip and RNA-seq data from this study have been submitted to the NCBI Gene Expression Omnibus (GEO; https://www.ncbi. nlm.nih.gov/geo/) under accession numbers GSE106499 and GSE104391, respectively.

\section{Acknowledgments}

We thank T. Rio Frio, S. Baulande, and P. Legoix-Né (NGS platform, Institut Curie); A. Lermine (bioinformatics platform, Institut Curie); and P. Lebaccon (PICT@Pasteur microscopy platform) for support. We thank Jacob Swadling and Alexander Esin for helping with the language. We also thank Camille Gautier for help with multiple mapping reads analysis and Marta Kwapisz for RNA-seq library preparation. We thank Valérie Borde for help with microarray experiments; David Sitbon, Valérie Borde, and Arnaud De Muyt for fruitful discussions; and David Sitbon and Ann EhrenhoferMurray for critical reading of the manuscript. We thank Layla El Mossadeq for her contribution to the construction of the SGA query strains. This work has benefited from the facilities and expertise of the NGS platform of Institut Curie (supported by the Agence Nationale de la Recherche [ANR-10-EQPX-03, ANR10-INBS-0908] and the Canceropôle Ile-de-France). The Morillon laboratory is supported by the Agence Nationale de la Recherche (DNA-life) and the European Research Council (EpincRNA starting grant, DARK consolidator grant). The Taddei laboratory is supported by funding from the Labex DEEP (ANR-11-LABX-0044_DEEP and ANR-10-IDEX-0001-02 PSL), from the ANR DNA-Life and DICENs (ANR-15-CE12-0007; ANR-14-CE10-0021-04), and Fondation pour la Recherche Médicale grant DEP20131128535. A.H. was supported by fellowships from the ENS PhD program and the FRM (FDT20160435654).

\section{References}

Altaf M, Utley RT, Lacoste N, Tan S, Briggs SD, Côté J. 2007. Interplay of chromatin modifiers on a short basic patch of histone $\mathrm{H} 4$ tail defines the boundary of telomeric heterochromatin. Mol Cell 28: 1002-1014. doi:10.1016/j.molcel.2007.12.002

Aparicio OM, Billington BL, Gottschling DE. 1991. Modifiers of position effect are shared between telomeric and silent mating-type loci in $S$. cerevisiae. Cell 66: 1279-1287. doi:10.1016/0092-8674(91)90049-5

Armache KJ, Garlick JD, Canzio D, Narlikar GJ, Kingston RE. 2011. Structural basis of silencing: Sir3 BAH domain in complex with a nucleosome at $3.0 \AA$ resolution. Science (80-) 334: 977-982. doi:10.1126/ science. 1210915

Arnaudo N, Fernández IS, McLaughlin SH, Peak-Chew SY, Rhodes D, Martino F. 2013. The N-terminal acetylation of Sir3 stabilizes its binding to the nucleosome core particle. Nat Struct Mol Biol 20: 1119-1121. doi: $10.1038 / \mathrm{nsmb} .2641$

Batté A, Brocas C, Bordelet H, Hocher A, Ruault M, Adjiri A, Taddei A, Dubrana K. 2017. Recombination at subtelomeres is regulated by physical distance, double-strand break resection and chromatin status. EMBO J 36: 2609-2625. doi:10.15252/embj.201796631

Behrouzi R, Lu C, Currie M, Jih G, Iglesias N, Moazed D. 2016. Heterochromatin assembly by interrupted Sir3 bridges across neighboring nucleosomes. eLife 5: e17556. doi:10.7554/eLife.17556

Bi X. 2002. Domains of gene silencing near the left end of chromosome III in Saccharomyces cerevisiae. Genetics 160: 1401-1407.

Bi X, Braunstein M, Shei GJ, Broach JR. 1999. The yeast HML I silencer defines a heterochromatin domain boundary by directional establishment of silencing. Proc Natl Acad Sci 96: 11934-11939. doi:10.1073/ pnas.96.21.11934

Bitterman KJ, Anderson RM, Cohen HY, Latorre-Esteves M, Sinclair DA. 2002. Inhibition of silencing and accelerated aging by nicotinamide, a putative negative regulator of yeast Sir2 and human SIRT1. J Biol Chem 277: 45099-45107. doi:10.1074/jbc.M205670200

Carmen AA, Milne L, Grunstein M. 2002. Acetylation of the yeast histone $\mathrm{H} 4 \mathrm{~N}$ terminus regulates its binding to heterochromatin protein SIR3. J Biol Chem 277: 4778-4781. doi:10.1074/jbc.M110532200

Choe W, Budd M, Imamura O, Hoopes L, Campbell JL. 2002. Dynamic localization of an Okazaki fragment processing protein suggests a novel role in telomere replication. Mol Cell Biol 22: 4202-4217. doi:10.1128/ MCB.22.12.4202-4217.2002

Dai J, Boeke JD. 2012. Strain construction and screening methods for a yeast histone H3/H4 mutant library. Methods Mol Biol 833: 1-14. doi:10.1007/978-1-61779-477-3_1

Deutschbauer AM, Jaramillo DF, Proctor M, Kumm J, Hillenmeyer ME, Davis RW, Nislow C, Giaever G. 2005. Mechanisms of haploinsufficiency revealed by genome-wide profiling in yeast. Genetics 169: 1915-1925. doi:10.1534/genetics.104.036871

Dion MF, Kaplan T, Kim M, Buratowski S, Friedman N, Rando OJ. 2007. Dynamics of replication-independent histone turnover in budding yeast. Science 315: 1405-1408. doi:10.1126/science.1134053

Donze D, Kamakaka RT. 2001. RNA polymerase III and RNA polymerase II promoter complexes are heterochromatin barriers in Saccharomyces cerevisiae. EMBO J 20: 520-531. doi:10.1093/emboj/20.3.520

Donze D, Kamakaka RT. 2002. Braking the silence: how heterochromatic gene repression is stopped in its tracks. BioEssays 24: 344-349. doi:10.1002/bies.10072

Donze D, Adams CR, Rine J, Kamakaka RT. 1999. The boundaries of the silenced HMR domain in Saccharomyces cerevisiae. Genes Dev 13: 698-708. doi:10.1101/gad.13.6.698

Ellahi A, Thurtle DM, Rine J. 2015. The chromatin and transcriptional landscape of native Saccharomyces cerevisiae telomeres and subtelomeric domains. Genetics 200: 505-521. doi:10.1534/genetics.115.175711 
Fourel G, Revardel E, Koering CE, Gilson E. 1999. Cohabitation of insulators and silencing elements in yeast subtelomeric regions. EMBO $J$ 18: 2522-2537. doi:10.1093/emboj/18.9.2522

Fourel G, Miyake T, Defossez PA, Li R, Gilson E. 2002. General regulatory factors (GRFs) as genome partitioners. J Biol Chem 277: 41736-41743. doi:10.1074/jbc.M202578200

Gartenberg MR, Smith JS. 2016. The nuts and bolts of transcriptionally silent chromatin in Saccharomyces cerevisiae. Genetics 203: 1563-1599. doi:10.1534/genetics.112.145243

Gotta M, Laroche T, Formenton A, Maillet L, Scherthan H, Gasser SM. 1996. The clustering of telomeres and colocalization with Rap1, Sir3, and Sir4 proteins in wild-type Saccharomyces cerevisiae. I Cell Biol 134: 1349-1363. doi:10.1083/jcb.134.6.1349

Grewal SI, Jia S. 2007. Heterochromatin revisited. Nat Rev Genet 8: 35-46. doi: $10.1038 / \operatorname{nrg} 2008$

Grunstein M. 1997. Molecular model for telomeric heterochromatin in yeast. Curr Opin Cell Biol 9: 383-387. doi:10.1016/S0955-0674(97) 80011-7

Grunstein M, Gasser SM. 2013. Epigenetics in Saccharomyces cerevisiae. Cold Spring Harb Perspect Biol 5: a017491. doi:10.1101/cshperspect.a017491

Guidi M, Ruault M, Marbouty M, Loïodice I, Cournac A, Billaudeau C, Hocher A, Mozziconacci J, Koszul R, Taddei A. 2015. Spatial reorganization of telomeres in long-lived quiescent cells. Genome Biol 16: 206. doi:10.1186/s13059-015-0766-2

Guillemette B, Bataille AR, Gévry N, Adam M, Blanchette M, Robert F, Gaudreau L. 2005. Variant histone H2A.z is globally localized to the promoters of inactive yeast genes and regulates nucleosome positioning. PLoS Biol 3: e384. doi:10.1371/journal.pbio.0030384

Guintini L, Tremblay M, Toussaint M, D'Amours A, Wellinger RE, Wellinger RJ, Conconi A. 2017. Repair of UV-induced DNA lesions in natural Saccharomyces cerevisiae telomeres is moderated by Sir2 and Sir3, and inhibited by yKu-Sir4 interaction. Nucleic Acids Res 45: 4577-4589. doi:10.1093/nar/gkx123

Harbison CT, Gordon DB, Lee TI, Rinaldi NJ, Macisaac KD, Danford TW, Hannett NM, Tagne JB, Reynolds DB, Yoo J, et al. 2004. Transcriptional regulatory code of a eukaryotic genome. Nature 431: 99-104. doi:10.1038/nature02800

Hecht A, Strahl-Bolsinger S, Grunstein M. 1996. Spreading of transcriptional repressor SIR3 from telomeric heterochromatin. Nature 383: 92-96. doi: $10.1038 / 383092 \mathrm{a} 0$

Heitz E. 1928. Das Heterochromatin der Moose. Jahrbücher für Wissenschaftliche Bot 69: 762-818.

Hesselberth JR, Chen X, Zhang Z, Sabo PJ, Sandstrom R, Reynolds AP, Thurman RE, Neph S, Kuehn MS, Noble WS, et al. 2009. Global mapping of protein-DNA interactions in vivo by digital genomic footprinting. Nat Methods 6: 283-289. doi:10.1038/nmeth.1313

Hoppe GJ, Tanny JC, Rudner AD, Gerber SA, Danaie S, Gygi SP, Moazed D. 2002. Steps in assembly of silent chromatin in yeast: Sir3-independent binding of a Sir2/Sir4 complex to silencers and role for Sir2dependent deacetylation. Mol Cell Biol 22: 4167-4180. doi:10.1128/ MCB.22.12.4167-4180.2002

Kasinathan S, Orsi GA, Zentner GE, Ahmad K, Henikoff S. 2014. High-resolution mapping of transcription factor binding sites on native chromatin. Nat Methods 11: 203-209. doi:10.1038/nmeth.2766

Katan-Khaykovich Y, Struhl K. 2005. Heterochromatin formation involves changes in histone modifications over multiple cell generations. EMBO J 24: 2138-2149. doi:10.1038/sj.emboj.7600692

Kemmeren P, Sameith K, Van De Pasch LAL, Benschop JJ, Lenstra TL, Margaritis T, O'Duibhir E, Apweiler E, Van Wageningen S, Ko CW et al. 2014. Large-scale genetic perturbations reveal regulatory networks and an abundance of gene-specific repressors. Cell 157: 740-752. doi:10.1016/j.cell.2014.02.054

Kitada T, Schleker T, Sperling AS, Xie W, Gasser SM, Grunstein M. 2011. $\gamma \mathrm{H} 2 \mathrm{~A}$ is a component of yeast heterochromatin required for telomere elongation. Cell Cycle 10: 293-300. doi:10.4161/cc.10.2.14536

Kitada T, Kuryan BG, Tran NN, Song C, Xue Y, Carey M, Grunstein M. 2012. Mechanism for epigenetic variegation of gene expression at yeast telomeric heterochromatin. Genes Dev 26: 2443-2455. doi:10.1101/ gad.201095.112

Kristjuhan A, Wittschieben BØ, Walker J, Roberts D, Cairns BR, Svejstrup JQ. 2003. Spreading of Sir3 protein in cells with severe histone $\mathrm{H} 3$ hypoacetylation. Proc Natl Acad Sci 100: 7551-7556. doi:10.1073/ pnas.1332299100

Kushnirov VV. 2000. Rapid and reliable protein extraction from yeast. Yeast 16: 857-860. doi:10.1002/1097-0061(20000630)16:9<857::AIDYEA561>3.0.CO;2-B

Lenstra TL, Benschop JJ, Kim T, Schulze JM, Brabers NA, Margaritis T, van de Pasch LA, van Heesch SA, Brok MO, Groot Koerkamp MJ, et al. 2011. The specificity and topology of chromatin interaction pathways in yeast. Mol Cell 42: 536-549. doi:10.1016/j.molcel.2011.03.026
Lieb JD, Liu X, Botstein D, Brown PO. 2001. Promoter-specific binding of Rap1 revealed by genome-wide maps of protein-DNA association. Nat Genet 28: 327-334. doi:10.1038/ng569

Louis EJ, Becker C, Marion M. 2014. Subtelomeres. Springer-Verlag, Berlin/ Heidelberg, Germany.

Marchfelder U, Rateitschak K, Ehrenhofer-Murray AE. 2003. SIR-dependent repression of non-telomeric genes in Saccharomyces cerevisiae? Yeast 20: 797-801. doi:10.1002/yea.1001

Martino F, Kueng S, Robinson P, Tsai-Pflugfelder M, van Leeuwen F, Ziegler M, Cubizolles F, Cockell MM, Rhodes D, Gasser SM. 2009. Reconstitution of yeast silent chromatin: multiple contact sites and O-AADPR binding load SIR complexes onto nucleosomes in vitro. Mol Cell 33: 323-334. doi:10.1016/j.molcel.2009.01.009

Martins-Taylor K, Sharma U, Rozario T, Holmes SG. 2011. H2A.Z (Htz1) controls the cell-cycle-dependent establishment of transcriptional silencing at Saccharomyces cerevisiae telomeres. Genetics 187: 89-104. doi:10.1534/genetics.110.123844

Matsuda A, Chikashige Y, Ding DQ, Ohtsuki C, Mori C, Asakawa H, Kimura H, Haraguchi T, Hiraoka Y. 2015. Highly condensed chromatins are formed adjacent to subtelomeric and decondensed silent chromatin in fission yeast. Nat Commun 6: 7753 . doi:10.1038/ncomms8753

Meneghini MD, Wu M, Madhani HD. 2003. Conserved histone variant H2A.Z protects euchromatin from the ectopic spread of silent heterochromatin. Cell 112: 725-736. doi:10.1016/S0092-8674(03)00123-5

Millar CB, Grunstein M. 2006. Genome-wide patterns of histone modifications in yeast. Nat Rev Mol Cell Biol 7: 657-666. doi:10.1038/nrm1986

Mitsumori R, Ohashi T, Kugou K, Ichino A, Taniguchi K, Ohta K, Uchida H, Oki M. 2016. Analysis of novel Sir3 binding regions in Saccharomyces cerevisiae. J Biochem 160: 11-17. doi:10.1093/jb/mvw021

Moazed D, Kistler A, Axelrod A, Rine J, Johnson AD. 1997. Silent information regulator protein complexes in Saccharomyces cerevisiae: a SIR2/ SIR4 complex and evidence for a regulatory domain in SIR4 that inhibits its interaction with SIR3. Proc Natl Acad Sci 94: 2186-2191. doi:10.1073/ pnas.94.6.2186

$\mathrm{Ng} \mathrm{HH}$, Feng Q, Wang H, Erdjument-Bromage H, Tempst P, Zhang Y, Struhl K. 2002. Lysine methylation within the globular domain of histone $\mathrm{H} 3$ by Dot 1 is important for telomeric silencing and Sir protein association. Genes Dev 16: 1518-1527. doi:10.1101/gad.1001502

Oki M, Valenzuela L, Chiba T, Ito T, Kamakaka RT. 2004. Barrier proteins remodel and modify chromatin to restrict silenced domains. Mol Cell Biol 24: 1956-1967. doi:10.1128/MCB.24.5.1956-1967.2004

Osborne EA, Dudoit S, Rine J. 2009. The establishment of gene silencing at single-cell resolution. Nat Genet 41: 800-806. doi:10.1038/ng.402

Park D, Lee Y, Bhupindersingh G, Iyer VR. 2013. Widespread misinterpretable ChIP-seq bias in yeast. PLoS One 8: e83506. doi:10.1371/journal pone.0083506

Pelechano V, Chávez S, Pérez-Ortín JE. 2010. A complete set of nascent transcription rates for yeast genes. PLoS One 5: e15442. doi:10.1371/journal. pone.0015442

Peng J, Zhou JQ. 2012. The tail-module of yeast Mediator complex is required for telomere heterochromatin maintenance. Nucleic Acids Res 40: $581-593$. doi: $10.1093 /$ nar/gkr757

Preti M, Ribeyre C, Pascali C, Bosio MC, Cortelazzi B, Rougemont J, Guarnera E, Naef F, Shore D, Dieci G. 2010. The telomere-binding protein Tbf1 demarcates snoRNA gene promoters in Saccharomyces cerevisiae. Mol Cell 38: 614-620. doi:10.1016/j.molcel.2010.04.016

Pryde FE, Louis EJ. 1999. Limitations of silencing at native yeast telomeres. EMBO J 18: 2538-2550. doi:10.1093/emboj/18.9.2538

$\mathrm{R}$ Core Team. 2016. $R$ : a language and environment for statistical computing. $\mathrm{R}$ Foundation for Statistical Computing, Vienna, Austria. https://www. R-project.org/

Radman-Livaja M, Ruben G, Weiner A, Friedman N, Kamakaka R, Rando OJ. 2011. Dynamics of Sir3 spreading in budding yeast: secondary recruitment sites and euchromatic localization. EMBO J 30: 1012-1026. doi:10.1038/emboj.2011.30

Renauld H, Aparicio OM, Zierath PD, Billington BL, Chhablani SK, Gottschling DE. 1993. Silent domains are assembled continuously from the telomere and are defined by promoter distance and strength, and by SIR3 dosage. Genes Dev 7: 1133-1145. doi:10.1101/ gad.7.7a.1133

Rhee HS, Pugh BF. 2011. Comprehensive genome-wide protein-DNA interactions detected at single-nucleotide resolution. Cell 147: 1408-1419. doi:10.1016/j.cell.2011.11.013

Rhee HS, Pugh BF. 2012. ChiP-exo method for identifying genomic location of DNA-binding proteins with near-single-nucleotide accuracy. Curr Protoc Mol Biol Chapter 21: Unit 21.24. doi:10.1002/0471142727. $\mathrm{mb} 2124 \mathrm{~s} 100$

Richards EJ, Elgin SCR. 2002. Epigenetic codes for heterochromatin formation and silencing: rounding up the usual suspects. Cell 108: 489-500. doi:10.1016/S0092-8674(02)00644-X

\section{Genome Research}

www.genome.org 
Rine J, Herskowitz I. 1987. Four genes responsible for a position effect on expression from $H M L$ and HMR in Saccharomyces cerevisiae. Genetics 116: 9-22.

Robinson MD, McCarthy DJ, Smyth GK. 2010. edgeR: a Bioconductor package for differential expression analysis of digital gene expression data. Bioinformatics 26: 139-140. doi:10.1093/bioinformatics/btp616

Robyr D, Suka Y, Xenarios I, Kurdistani SK, Wang A, Suka N, Grunstein M. 2002. Microarray deacetylation maps determine genome-wide functions for yeast histone deacetylases. Cell 109: 437-446. doi:10.1016/ S0092-8674(02)00746-8

Roy R, Meier B, McAinsh AD, Feldmann HM, Jackson SP. 2004. Separationof-function mutants of yeast Ku80 reveal a Yku80p-Sir4p interaction involved in telomeric silencing. J Biol Chem 279: 86-94. doi:10.1074/jbc. M306841200

Ruault M, De Meyer A, Loïodice I, Taddei A. 2011. Clustering heterochromatin: Sir3 promotes telomere clustering independently of silencing in yeast. J Cell Biol 192: 417-431. doi:10.1083/jcb.201008007

Rudner AD, Hall BE, Ellenberger T, Moazed D. 2005. A nonhistone proteinprotein interaction required for assembly of the SIR complex and silent chromatin. Mol Cell Biol 25: 4514-4528. doi:10.1128/MCB.25.11.45144528.2005

Rusche LN, Kirchmaier AL, Rine J. 2003. The establishment, inheritance, and function of silenced chromatin in Saccharomyces cerevisiae. Annu Rev Biochem 72: 481-516. doi:10.1146/annurev.biochem.72.121801. 161547

Schulze JM, Jackson J, Nakanishi S, Gardner JM, Hentrich T, Haug J, Johnston M, Jaspersen SL, Kobor MS, Shilatifard A. 2009. Linking cell cycle to histone modifications: SBF and H2B monoubiquitination machinery and cell-cycle regulation of H3K79 dimethylation. Mol Cell 35: 626-641. doi:10.1016/j.molcel.2009.07.017

Shampay J, Szostak JW, Blackburn EH. 1984. DNA sequences of telomeres maintained in yeast. Nature 310: $154-157$. doi:10.1038/310154a0

Sperling AS, Grunstein M. 2009. Histone H3 N-terminus regulates higher order structure of yeast heterochromatin. Proc Natl Acad Sci 106: 1315313159. doi:10.1073/pnas.0906866106

Strahl-Bolsinger S, Hecht A, Luo K, Grunstein M. 1997. SIR2 and SIR4 interactions differ in core and extended telomeric heterochromatin in yeast. Genes Dev 11: 83-93. doi:10.1101/gad.11.1.83

Stulemeijer IJ, De Vos D, van Harten K, Joshi OK, Blomberg O, van Welsem T, Terweij M, Vlaming H, de Graaf EL, Altelaar AF, et al. 2015. Dot1 histone methyltransferases share a distributive mechanism but have highly diverged catalytic properties. Sci Rep 5: 9824 . doi:10.1038/srep.09824

Suka N, Luo K, Grunstein M. 2002. Sir2p and Sas2p opposingly regulate acetylation of yeast histone H4 lysine 16 and spreading of heterochromatin. Nat Genet 32: 378-383. doi:10.1038/ng1017

Szilard RK, Jacques PE, Laramée L, Cheng B, Galicia S, Bataille AR, Yeung M, Mendez M, Bergeron M, Robert F, et al. 2010. Systematic identification of fragile sites via genome-wide location analysis of $\gamma$-H2AX. Nat Struct Mol Biol 17: 299-305. doi:10.1038/nsmb.1754
Takahashi YH, Schulze JM, Jackson J, Hentrich T, Seidel C, Jaspersen SL, Kobor MS, Shilatifard A. 2011. Dot1 and histone H3K79 methylation in natural telomeric and HM silencing. Mol Cell 42: 118-126. doi:10.1016/j.molcel.2011.03.006

Talbert PB, Henikoff S. 2006. Spreading of silent chromatin: inaction at a distance. Nat Rev Genet 7: 793-803. doi:10.1038/nrg1920

Teytelman L, Thurtle DM, Rine J, van Oudenaarden A. 2013. Highly expressed loci are vulnerable to misleading ChIP localization of multiple unrelated proteins. Proc Natl Acad Sci 110: 18602-18607. doi:10.1073/ pnas. 1316064110

Thurtle DM, Rine J. 2014. The molecular topography of silenced chromatin in Saccharomyces cerevisiae. Genes Dev 28: 245-258. doi:10.1101/ gad.230532.113

Tong AH, Evangelista M, Parsons AB, Xu H, Bader GD, Pagé N, Robinson M, Raghibizadeh S, Hogue CW, Bussey H, et al. 2001. Systematic genetic analysis with ordered arrays of yeast deletion mutants. Science 294: 2364-2368. doi:10.1126/science.1065810

Tsukamoto Y, Kato JI, Ikeda H. 1997. Silencing factors participate in DNA repair and recombination in Saccharomyces cerevisiae. Nature 388: 900-903. doi:10.1038/42288

Van De Vosse DW, Wan Y, Lapetina DL, Chen WM, Chiang JH, Aitchison JD, Wozniak RW. 2013. A role for the nucleoporin Nup170p in chromatin structure and gene silencing. Cell 152: 969-983. doi:10.1016/ j.cell.2013.01.049

Wagih O, Usaj M, Baryshnikova A, VanderSluis B, Kuzmin E, Costanzo M, Myers CL, Andrews BJ, Boone CM, Parts L. 2013. SGAtools: one-stop analysis and visualization of array-based genetic interaction screens. Nucleic Acids Res 41: W591-W596. doi:10.1093/nar/gkt400

Wang F, Li G, Altaf M, Lu C, Currie MA, Johnson A, Moazed D. 2013. Heterochromatin protein Sir3 induces contacts between the amino terminus of histone $\mathrm{H} 4$ and nucleosomal DNA. Proc Natl Acad Sci 110: 8495-8500. doi:10.1073/pnas.1300126110

Wang J, Jia ST, Jia S. 2016. New insights into the regulation of heterochromatin. Trends Genet 32: 284-294. doi:10.1016/j.tig.2016.02.005

Weiner A, Hsieh TH, Appleboim A, Chen HV, Rahat A, Amit I, Rando OJ, Friedman N. 2015. High-resolution chromatin dynamics during a yeast stress response. Mol Cell 58: 371-386. doi:10.1016/j.molcel.2015.02. 002

Yu MC, Lamming DW, Eskin JA, Sinclair DA, Silver PA. 2006. The role of protein arginine methylation in the formation of silent chromatin. Genes Dev 20: 3249-3254. doi:10.1101/gad.1495206

Yue JX, Li J, Aigrain L, Hallin J, Persson K, Oliver K, Bergström A, Coupland P, Warringer J, Lagomarsino MC, et al. 2017. Contrasting evolutionary genome dynamics between domesticated and wild yeasts. Nat Genet 49: $913-924$. doi:10.1038/ng.3847

Received March 6, 2018; accepted in revised form October 20, 2018. 


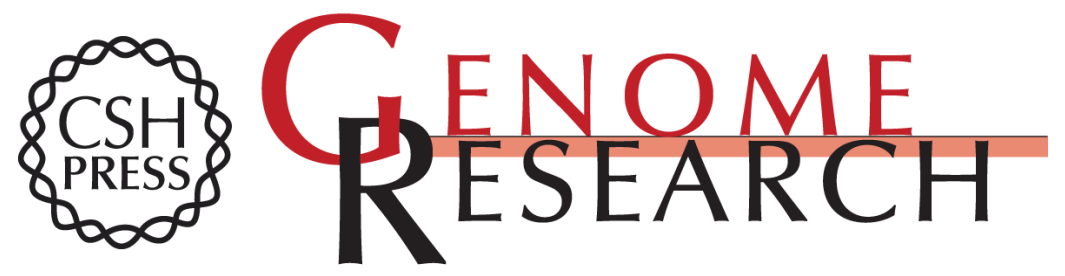

\section{Expanding heterochromatin reveals discrete subtelomeric domains delimited by chromatin landscape transitions}

Antoine Hocher, Myriam Ruault, Petra Kaferle, et al.

Genome Res. 2018 28: 1867-1881 originally published online October 24, 2018

Access the most recent version at doi:10.1101/gr.236554.118

Supplemental Material

References

Creative

Commons

License

Email Alerting

Service
http://genome.cshlp.org/content/suppl/2018/11/15/gr.236554.118.DC1

This article cites 90 articles, 36 of which can be accessed free at: http://genome.cshlp.org/content/28/12/1867.full.html\#ref-list-1

This article is distributed exclusively by Cold Spring Harbor Laboratory Press for the first six months after the full-issue publication date (see

$\mathrm{http}: / / g$ enome.cshlp.org/site/misc/terms.xhtml). After six months, it is available under a Creative Commons License (Attribution-NonCommercial 4.0 International), as described at http://creativecommons.org/licenses/by-nc/4.0/.

Receive free email alerts when new articles cite this article - sign up in the box at the top right corner of the article or click here.

\section{Affordable, Accurate Sequencing.}

To subscribe to Genome Research go to:

https://genome.cshlp.org/subscriptions 